\title{
Integrated optical devices for lab-on-a-chip biosensing applications
}

\author{
M.-Carmen Estevez, Mar Alvarez and Laura M. Lechuga* \\ Nanobiosensors and Bioanalytical Applications Group, Research Center on \\ Nanoscience and Nanotechnology (CSIC) \& CIBER-BBN. Campus UAB, 08193 \\ Bellaterra, Barcelona, Spain
}

*Email: Laura.lechuga@cin2.es Phone: +34-935868012

Fax: +34-935868020

\begin{abstract}
The application of portable, easy-to-use and highly sensitive lab-on-achip biosensing devices for real-time diagnosis could offer significant advantages over current analytical methods. Integrated optics-based biosensors have become the most suitable technology for lab-on-chip integration due to their ability for miniaturization, their extreme sensitivity, robustness, reliability, and their potential for multiplexing and mass production at low cost. This review provides an extended overview of the state-of-the-art in integrated photonic biosensors technology including interferometers, grating couplers, microring resonators, photonic crystals and other novel nanophotonic transducers. Particular emphasis has been placed on describing their real biosensing applications and wherever possible a comparison of the sensing performances between each type of device is included. The way towards achieving operative lab-on-a-chip platform incorporating the photonic biosensors is also reviewed. Concluding remarks regarding the future prospects and potential impact of this technology are also provided.
\end{abstract}

Short title: M.C. Estevez, M. Alvarez and L.M. Lechuga: Integrated optical biosensors.

PACS: 87.85.fk, 42.82.Et, 07.07.Df, 07.07.Mp, 07.60.Ly, 87.85.Rs

Keywords: Photonic biosensors, evanescent wave detection, lab-on-a-chip, biofunctionalization, silicon photonics. 


\section{Introduction}

Biosensors are devices able to detect a specific substance by converting the recognition from a biological entity (i.e. DNA, antibody, enzyme,...) into an electrical signal that can be further processed and related to the concentration of the substance under analysis. Biosensors can provide selective, sensitive, fast, direct and cost-effective analyses. In addition, they can perform tests in real-time without using fluorescent labels or amplification steps and with a minimum volume of samples and reagents [1]. As compared to standard techniques which are usually time-consuming, expensive and require labelling and trained personal, biosensing technology offers clear advantages.

Lab-on-a-chip (LOC) are miniaturized devices in which all functionalities are integrated in the same platform, from sample preparation to signal delivery [2]. Ideally a LOC device should contain enough hard wired intelligence and robustness to be used by non-skilled personal and should deliver the results directly to a central monitoring station. It is clear that achieving a small, portable and easy-to-use lab-on-a-chip device for diagnostics could offer significant advantages over standard methods. Although significant progress has been accomplished at the LOC field during last years, very few stand-alone devices have emerged [3]. Most of current devices are simple planar microfluidic devices which do not incorporate the detection and after the reaction has taken place, the read-out must be done with complex instrumentation in laboratory settings. That is the main reason why incorporating "on-chip" detection by using biosensors is a new technology that shows great promise. Main application fields of this technology can be clinical diagnostics, environmental monitoring, chemical and biological warfare surveillance, food industry and veterinary and industrial process control, among others. By using this advanced technology, diagnosis in developing countries could become an important achievement for the near future.

Photonic biosensors are well-established technologies for the sensitive monitoring of molecular interactions. They could afford the requirements for the "on-chip" detection in lab-on-a-chip platforms due to their outstanding characteristics of sensitivity, label-free and real-time detection. The detection principle of most optical biosensors is based on the evanescent field detection. In the evanescent wave mechanism (see Figure 1), a bioreceptor layer is immobilized onto the surface of a waveguide; the exposure to the partner analyte produces a biomolecular interaction affecting the guiding properties of the waveguide (concretely, a variation of the refractive index) via the modification through the evanescent field. The variation of the refractive index can be evaluated by any of the waveguiding optical properties (intensity, phase, 
resonant momentum, polarization,...) and this variation can be correlated with the concentration of the analyte and with the affinity constant of the interaction, resulting in a quantitative value of the interaction.

As the evanescent wave decays exponentially while it penetrates into the outer medium, it only detects changes taking place on the surface of the waveguide, since the intensity of the evanescent field is much higher in this particular region. For most waveguide systems, this decay length is on the order of 0.1-1 $\mu \mathrm{m}$. For that reason, it is not necessary to carry out a prior separation of non-specific components (as in conventional analyses) because any change in the bulk solution will hardly affect the sensor response. Therefore, the most significant advantages of evanescent-based mechanism are the highly sensitive and specific label-free detection of target molecules or biochemical reactions in real time, with reduced nonspecific binding, which makes this detection mechanism one of the most useful for detection of targets in complex real samples.

The most common optical evanescent wave biosensor is the Surface Plasmon Resonance (SPR) device [4] based on the variation of the reflectivity on a metallic layer in close contact with a dielectric media. The SPR biosensor has been widely developed and commercialized and hundreds of publications have demonstrated its outstanding performance to evaluate complex biosensing interactions [4]. But SPR sensor has a relatively large size and its miniaturization in lab-on-chip platforms is complex. Moreover, the sensitivity is usually limited to the nanomolar range, which is extremely useful in diverse applications [5-7] but not enough for applications requiring lower detection levels (pM-fM or even single-molecule), which are usual in the clinical practice.

Photonic sensors based on integrated optics (IO) could solve the aforementioned SPR problems, as they can be easily miniaturized and they offer high potential for chip integration; moreover, by using evanescent wave as detection mechanism, sensitivities can be extremely high (pM in a label-free scheme). Moreover, integrated optics allows a great flexibility in the materials and structures selection and fabrication of arrays of sensors with the same characteristics within the same chip for multiplexing analysis can be afforded. Materials employed in IO devices are Si, $\mathrm{Si}_{3} \mathrm{~N}_{4}, \mathrm{SiON}, \mathrm{SiO}_{2}$ or polymers, and techniques such as ion-diffusion in glass, chemical vapour deposition, spin-coating, nanoimprinting, electron beam lithography, etc are commonly employed for the fabrication. By using silicon photonics technology, additional advantages such as robustness, reliability, low power consumption and potential for mass production with consequent reduction of production costs are added. Other technologies as III-V or lithium 
niobate, often used in optical telecommunications, have shown less suitability for IO biosensors as they are more complex and expensive.

Most of the refractive index IO sensors rely on the induced changes of the effective refractive index by the biomolecular interaction in the evanescent area. In order to achieve highest sensitivity, the waveguiding structure must be optimized looking for a maximum change of the effective refractive index due to the sensing biolayer. Sensitivity is a complex parameter involving geometry, material and working wavelength of the waveguide, and also other aspects such as the chemical activation of the surface, the biofunctionalization method and the resolution and noise of the optical read-out system. In addition, fluidics must be taken into account (i.e. flow cell volume, way of sample injection, diffusion, dispersion). Each of these aspects must be properly designed and optimized before delivering a functional biosensor device. Integrated optical sensors such as grating-couplers, interferometers, photonic crystal, microring resonators, slot waveguides or silicon wires have been extensively studied in the last years [8-12], but so far only few of them are commercially available.

One of the main advantages of the IO technology is the possibility to integrate all the functions (chemical, optical, microfludics and electronics) in one single platform offering an ideal solution for the implementation of truly lab-on-a-chip devices. This area is still in its infancy, but remarkable progress has been achieved during last years $[3,13]$. This is reflected in the increasing amount of publications addressing new or optimized sensing configurations.

In this chapter, an overview of the main integrated optical sensors will be presented with special focus on the most relevant ones in terms of sensing performance and integration capability, and whose feasibility for label-free biosensing has been proven. For each of them, a brief description of its operating principle, design, fabrication and read-out resolution will be presented, and, when reported, the biosensing dynamic range and detection limit will be summarized. Application in clinical diagnostics, environmental monitoring, or in food and agroalimentary industry will be included as well as a brief section describing the commercial devices already on the market. One of the crucial aspects that will be discussed is the way towards a truly lab-on-achip integration, although few examples of completely integrated platforms can be found in the literature. Finally, an outlook of the future prospects of this technology will be discussed.

\section{Biofunctionalization and immobilization strategies}

The selection of an appropriate procedure to immobilize the biological element on the sensor surface has become a critical step in the biosensor area, and enormous efforts are continuously invested in order to optimize novel strategies according with the application. The 
immobilization process should not only guarantee an efficient coverage of the transducer surface with the biomolecules, while keeping intact their properties (functionality, structure, biological activity, affinity, specificity...), but it should also ensure their stability for storage and regeneration. Moreover, most of the applications, such as clinical and medical diagnosis, agroalimentary analysis, or environmental field surveillance, require well-defined surfaces with biocompatible properties, minimizing non-specific adsorption when analyzing complex real samples. The choice of the most effective strategy of immobilization which combines the above considerations usually becomes the key factor which turns a sensing device into a valid and applicable analytical tool with the required quality standards. Moreover, the bioreceptor layer directly affects the reproducibility, selectivity and resolution of any sensor device.

A wide variety of biomolecules can be used as bioreceptors, i.e. antibodies, nucleic acid sequences, peptides, enzymes, cell receptors and many others. The selected biomolecule is dictated by the application and must be chosen to be highly specific for the target molecule and stable enough to be immobilized without losing functionality. Several types of routes can be used to biofunctionalize the sensor surface: (i) physical adsorption by direct deposition of the biomolecule; (ii) covalent binding of the biomolecule to the surface (using a cross-linker previously immobilized on the surface or following more complex strategies [14]); (iii) noncovalent interactions to a previously deposited active layer, either by non-specific electrostatic interactions or by non-covalent affinity binding (i.e. biotin-avidin systems, His-Tag system, Protein $\mathrm{A} / \mathrm{G}$ for antibodies) (iv) physical entrapment in a polymer layer. Figure 2 summarizes the general immobilization strategies.

Physical adsorption is a simple strategy based on hydrophobic and electrostatic interactions between the biomolecule and the surface, but it can lead to the easy desorption of the active receptors under flow conditions and also when regeneration cocktails are applied to break the interaction event (which usually implies high or low $\mathrm{pH}$ solutions, salt concentrations, organic solvents, etc.). Moreover, issues related to reproducibility together with undesired folding of the biomolecules onto the surface are common drawbacks of this strategy which makes it not advisable in most of the cases even despite its simplicity.

Covalent binding can be made through one of the chemical groups of the biomolecule. It is recommended to use a group whose blocking does not compromise the overall functionality of the biomolecule. Amino, carboxylic or thiol groups are the preferred option to couple proteins. For nucleic acids immobilization, it is possible to take advantage of the versatility of the DNA synthesis which allows the incorporation of reactive groups at the end of the sequence. Specially difficult is the attachment of antibodies in an oriented way (leaving the affinity binding sites 
free), which can be done by controlled binding through the carbohydrates groups of the constant region or by using affinity proteins (such as A or G Protein) [15]. Another non-covalent affinity binding strategy is based on the biotin-avidin system, through the formation of a sandwich-like layer (biotin on the surface/avidin/biotinylated biomolecule) [14-16].

Before the biofunctionalization step, a previous chemical activation of the sensor surface is always needed. Silicon, silicon oxide and silicon nitride are conventional materials commonly used for integrated optical transducers which can be functionalized using the well-known silane chemistry. Silicon-based surfaces require a prior activation step to oxidize the surface and to expose the silanol groups for crosslinking with the silane. Whereas silicon oxide is hydrophilic, silicon nitride is an electronically neutral non-porous material, whose modification is usually not straightforward. In the case of silicon nitride, methods are based on an initial etching to remove the native $\mathrm{SiO}_{2}$ layer to further be oxidized to form a new oxide layer [17]. Other strategies involve the derivatization of the $\mathrm{Si}-\mathrm{H}$ and $\mathrm{Si}-\mathrm{N}$ groups of the silicon nitride using more drastic conditions [18-21]. The activated surface is subsequently modified with organosilanes forming a silane layer with exposed reactive groups susceptible to react with the bioreceptor. Hundreds of different organosilanes with a wide variety of structures, length, functionality and chemical and physical properties are nowadays commercially available, although the most commonly employed are those with short alkyl chain (i.e. propyl, butyl), ending in amino, thiol, epoxy or carboxylic groups.

As a first step, evaluation of the biosensor capability is done using purified or spiked samples in buffer. But in order to assess the real performance of any device, complex samples which contain high concentration of non-specific molecules, such as blood, urine, serum, saliva, tears, cerebrospinal or medullar fluids should be tested. For preparation of the receptor layer which must avoid matrix effects and large background signals, less conventional reagents that provide a more hydrophilic and biocompatible and antifouling surface are increasingly being used such as dextran polymer [22] or poly(ethylene) glycol (PEG) derivatives [23-28].

Overall, to achieve the full potential of the optical sensing devices, the choice of the surface modification, the biofunctionalization procedures, and the type and conditions of the assay become as relevant and crucial as the development and optimization itself of the integrated optics-based sensing structures.

\section{Biosensors based on Integrated Optics}


In this Section, an overview of the main biosensing technologies based on integrated optics will be presented. The main driving force behind the development of integrated optical biosensors is to push the sensitivity for label-free detection of minimum amounts of substances, which are the concentration normally found in human fluids at the starting of a disease (as cancer) or in contaminated water or food or in a biowarfare attack: the lower the limit of detection, the earlier the disease or the pollutant could be detected. Limit of detection (LOD) and sensitivity are the most important parameters which define the sensor performance. The sensitivity is mostly referred to the strength of light-matter interaction. Limit of detection can be defined as the smallest amount of analyte that produces a quantifiable output signal and will depend on the resolution of the optical read-out system and therefore it is strongly related to the experimental noise. Contributions from laser or light source intensity fluctuations, microfluidics, and thermal variations, among others, can have a strong effect on the overall noise. Thus, while the sensitivity of a device can be very high, the associated noise can lead to moderate LOD. Moreover, for biosensing a receptor must be previously immobilized, and the biofunctionalization protocol plays also a crucial role in achieving low detection limits. We have chosen the limit of detection as the most suitable parameter for comparing the different IO technologies. Two main ways of specifying the limit of detection can be employed: (i) according to the bulk sensitivity (expressed as refractive index units (RIU) as all the evanescent wave sensors are sensitive to any change in the bulk refractive index of the solution above the sensor surface; (ii) according to the surface sensitivity, as the evanescent sensors are sensitive to any accumulation of mass on the sensor surface, which is an evaluation of the real biosensing capabilities of a transducer, normally expressed as surface mass density $\left(\mathrm{pg} / \mathrm{mm}^{2}\right)$. Detection limit can also be expressed as analyte concentration (i.e. in $\mathrm{ng} / \mathrm{mL}$ or molarity), but this value is not directly comparable among different sensors since it will depend on the target molecule and its affinity constant. Best resolution for bulk refractive index changes (bulk sensitivity) are within the range of $10^{-5}$ to $10^{-8}$ RIU, which depending on the analyte and transducer mechanism means that concentration down to $\mathrm{ng} / \mathrm{mL}$ or $\mathrm{pg} / \mathrm{mL}$ can be determined.

Table 1 shows a comparison of the limits of detection (in RIU and $\mathrm{pg} / \mathrm{mm}^{2}$ ) for the integrated optical sensors described along this review.

\subsection{Interferometric waveguide sensors}

Among the different integrated optical sensors, the interferometric ones are the most attractive for biosensing due to their high sensitivity and broad dynamic range. Mach-Zehnder (MZI), Young (YI) and Hartman interferometers are usual configurations employed for sensing (see Figure 3). 
In the integrated version of MZI, an input optical waveguide is split into two arms which after a certain distance are recombined again in an output optical waveguide (see Figure 3.A. for general structure and 3.B for a view of an integrated array of MZI). A biomolecular interaction in the sensor area within the evanescent field will produce a variation in the effective refractive index of the light propagating through this area, inducing a phase difference between the light travelling in the sensor and the reference arms. The interferometric modulation at the device output is described by:

$$
\begin{aligned}
& I=\frac{I_{0}}{2}\left[E_{S}^{2}+E_{R}^{2}+2 E_{S} E_{R} \cos \Delta \Phi\right] \\
& \Delta \Phi=\frac{2 \pi L}{\lambda}\left[n_{e f f, S}-n_{e f f, R}\right]
\end{aligned}
$$

Where $E$ is the electric field propagating along the waveguide, $n_{e f f}$ is the effective refractive index, $\Phi$ is the phase, $\mathrm{L}$ is the detection length, $\lambda$ is the light wavelength and $I_{0}$ and $I$ are the light intensity at the input and output, respectively. The labels $\mathrm{S}$ and $\mathrm{R}$ stands for the sensing and the reference arm, respectively. An attractive aspect of this device is the possibility of using long interaction lengths, increasing the sensitivity. The optimization of the signal-to-noise ratio implies maximizing the transfer functions and hence maximizing all partial sensitivities (as could be $\partial \Delta \Phi / \partial n_{\text {eff }}$, etc) and, at the same time, minimizing all the perturbing effects, as could be temperature or wavelength drift. As can be deduced from equation (1), one main drawback of the interferometric sensors is that due to the cosine dependency, the sensitivity will depend on the position of the interferometric curve, with higher sensitivity at the quadrature points and strongly decreasing near the maximum or minimum of the curve. Modulation system should be an option in order to track the response to the quadrature position.

The main condition of an integrated interferometric device for biosensing application is the single mode behaviour of the waveguides. Each mode in a waveguide propagates at a different speed and its evanescent tail is different. Hence, if several modes, each one having a different sensitivity, simultaneously propagate in the waveguide, the information carried by them interferes, resulting in a decrease of the signal. For a fixed working wavelength, the modal properties of a guiding structure depend on the thickness of the waveguide core and on the refractive index contrast between the core and the surrounding media (claddings). To obtain single-mode waveguides, it is required to have a minimum difference between the refractive indices (RI) and/or a decrease of the waveguide dimensions. As a rule, if the difference between the RI of the core and the cladding is higher than $10 \%$, single-mode can only be achieved with core thicknesses of hundreds of nanometers. In addition, for dense packaging of devices or for defining complex devices (as Mach-Zehnder interferometers), the waveguides must have 2D- 
confinement, that is, light should remain confined in the cross-section. There are several possibilities to achieve this confinement, but the most commonly employed is to partially etch the waveguide core forming a rib which confines the light in the transversal direction. A careful design of the rib is required to avoid excitation of lateral modes. As an example, for high index contrast waveguides (as for example $\mathrm{Si}_{3} \mathrm{~N}_{4} / \mathrm{SiO}_{2}$ ), the rib should not exceed several nanometers in depth to assure single mode behavior.

Glass-based [29-32] and polymer-based MZ interferometers using NOA81 [33] or SU-8 resist [34], have been fabricated, and even assessed for biosensing purposes [35] with promising results. Recently, Crespi et al. have reported the fabrication of a 3D MZI inscribed in a fused silica chip with a previous fabricated microchannel, by using femtosecond laser writing [32]. However, in spite of the compactness and the high integration reached, the extrapolated LOD is only of $1.5 \cdot 10^{-4} \mathrm{RIU}$, and no biosensing results have been reported. Most common MZ interferometers are based on silicon nitride as core layer and silica as cladding. Initial works with silicon-based MZI were addressed to optimize the structure and to improve the sensitivities for biosensing applications [36-40]. Two different configurations, one based on total internal reflection (TIR-based MZI) [37,41] and another one based on anti-resonant reflecting optical waveguide (ARROW-based MZI) [42,43] have been proposed.

For TIR waveguides using silicon-related materials, maximum surface sensitivity and single mode behavior are achieved for high refractive index contrast and core thicknesses of hundreds of nanometers. The reported sensor has a core waveguide layer of $250 \mathrm{~nm}\left(\mathrm{Si}_{3} \mathrm{~N}_{4}, \mathrm{n}=2.00\right)$, a width of $4 \mu \mathrm{m}$ and a rib of $3 \mathrm{~nm}$, over a $\mathrm{SiO}_{2}(\mathrm{n}=1.46)$ cladding layer of $2 \mu \mathrm{m}$. The device is covered with a protective $\mathrm{SiO}_{2}$ layer except for the sensor area, to bring the waveguide into contact with the environment [41]. Bulk sensitivity for a device with a sensor window of $15 \mathrm{~mm}$ showed a limit of detection of refractive index of $8 \cdot 10^{-6}$ RIU , which corresponds to a surface sensitivity of around $2 \cdot 10^{-4} \mathrm{~nm}^{-1}$. Preliminary biosensing measurements based on antigenantibody interactions showed the feasibility of the device. Further biosensing real time experiments of DNA hybridization reached detection levels of target complementary DNA of 10 pM with, high specificity as compared to control sequences [44] which means an extremely high surface mass LOD of $0.06 \mathrm{pg} / \mathrm{mm}^{2}$.

An ARROW-based MZI was also developed [43]. In this case, the optical confinement of light is based on antiresonant reflections rather than total internal reflections; these waveguides exhibit low losses and permit larger dimensions (micrometers instead of nanometers) for achieving single mode behaviour. An optimized structure with a core layer $\left(\mathrm{SiO}_{2},>2 \mu \mathrm{m}\right.$ thickness, $\left.\mathrm{n}_{\text {core }}=1.485\right)$, a second cladding layer $\left(\mathrm{SiO}_{2}, 2 \mu \mathrm{m}, \mathrm{n}=1.46\right)$ and a first cladding layer 
$\left(\mathrm{Si}_{3} \mathrm{~N}_{4}, 120 \mu \mathrm{m}\right.$ thickness, $\left.\mathrm{n}=2.00\right)$ was fabricated. The rib depth was $60 \%$ of the core thickness and the waveguide width should have to be lower than $8 \mu \mathrm{m}$ to obtain single-mode behavior [45]. Initial refractive index detection limit of $2.5 \cdot 10^{-5} \mathrm{RIU}$ was obtained [45], further improved to $2.5 \cdot 10^{-6} \mathrm{RIU}$ [46]. The device was also evaluated as immunosensor for the detection of an insecticide, carbaryl, by using a competitive immunoassay [46].

Young Interferometer (YI) is another well-explored interferometric device for biosensing. The YI is a waveguide with an integrated Y-junction acting as a beam splitter and, contrary to the MZI, the two beams are not recombined again. The exiting light from both arms is projected onto a CCD camera giving an interference pattern. When a biomolecular interaction takes place on the sensing arm, it induces a variation in the effective refractive index in this arm respect to the reference one $\left(n_{\text {eff, }, S}-n_{\text {eff, },}\right)$. The phase difference of the two interfering rays is given by:

$$
\Phi=\frac{2 \pi}{\lambda}\left(\frac{d \cdot x}{f}-\left(n_{e f f, S}-n_{e f f, R}\right) \cdot L\right)
$$

where $d$ is the distance between the two branches, $f$ is the distance between the output sensor and the read-out camera and $x$ denotes the position on the camera. As an output, the fringe pattern moves laterally. One disadvantage of the YI device is the distance required from the output to the detector in order to get a maximum resolution. Advantages of the YI include the simplicity of the arrangement, the detection of the complete intensity distribution and the identical length of the arms which avoids side effects arising from temperature and wavelength drifts.

Brandenburg et al. [47,48] developed a free-space YI based on $154 \mathrm{~nm}$ thick $\mathrm{Ta}_{2} \mathrm{O}_{5}$ waveguide with single mode behavior. In this configuration, two separate beams are coupled into the sensing chip via a grating coupler. After propagating through the channels, light from both beams are coupled out by a second grating and diffracted by a double slit. The interference pattern is monitored on a CCD camera. A detection limit based on adsorbed molecules on the surface reached values of $0.75 \mathrm{pg} / \mathrm{mm}^{2}$ and a resolvable variation of effective refractive index of $9 \cdot 10^{-8}$ RIU [47]. Using this device, two different monoclonal antibodies have been immobilized on both channels and the specificity has been monitored together with the influence of samples diluted in complex matrices such as blood [49]. A careful study of an immobilization procedure based on the non-covalent deposition of bilayers of antibodies was followed, which guaranteed the absence of adsorption of interfering substances when spiked blood samples were added. Although the sensitivity levels were not studied in depth, it was possible to detect a low molecular weight substance as methotrexate in a direct way. By incorporating a two-channel fluidic cell ( $3 \mu \mathrm{L}$ volume) for the reference and the sensing arm, the monitoring of recombinant 
protein production was attempted [50]. A methodology based on the immobilization onto a streptavidin modified surface of biotinylated antibodies, which recognize a tag introduced during the protein expression process, was carried out showing a LOD around $50 \mathrm{ng} / \mathrm{mL}$. This LOD slightly increases up to $350 \mathrm{ng} / \mathrm{mL}$ when cell lysate was used instead of buffer (as this is the real media from which the produced protein is extracted). Moreover, the viability of the device for clinical diagnostics was demonstrated by the detection of tuberculosis-specific antibodies in undiluted serum samples from infected patients [51]. A YI based on $\mathrm{Ta}_{2} \mathrm{O}_{5}$ planar waveguide (150 nm thick) with integrated gratings for in and out coupling of the light, and with a PDMS flow cell mounted on top, showed bulk detection limit of $5 \cdot 10^{-6}$ RIU and DNA hybridization sensing of $50 \mathrm{nM}$ [52].

Ymeti et al. have developed a four-channel integrated Young interferometer [53] that enables direct independent measurements in each sensing area, including one taken as a reference (Figure 3.C). The refractive index resolution was close to $8.5 \times 10^{-8} \mathrm{RIU}$ (corresponding to an estimated protein mass coverage resolution of $20 \mathrm{fg} / \mathrm{mm}^{2}$ ) [54] which is one of the most sensitive values so far reported. With this design it was possible to correct and reduce by ten times the temperature or setup drift. Moreover, the integration with microfluidics has reduced the sample volume and the analysis response time, opening up the possibility to have a miniaturized system. An immunoassay based on the immobilization of specific antibodies for herpes simplex virus type 1 (HSV-1) detection was implemented with this device. Using one channel as reference and the other one with an oriented antibody immobilization using protein A, a limit of detection as low as 850 particles $/ \mathrm{mL}$ was achieved, worsening when serum samples were evaluated $[55,56]$.

In the Hartman interferometer (Figure 3.D) a linearly polarized light is incoupled through multiple sensing areas of an array of interferometers by using a grating fabricated onto the chip. The viability of the platform for biosensing [57] was demonstrated by detecting levels of hormones as low as $0.5 \mathrm{ng} / \mathrm{mL}$ in undiluted serum and blood samples, using an amplified sandwich immunoassay $[58,59]$. An improved and more robust prototype integrating the laser diode, the waveguides, the flow cell and the CCD camera was developed. The device showed a LOD of $10^{-6}$ RIU and was recently used for the detection of avian influenza [60] by determining the virus antigen (HA; hemagluttination antigen) with the specific antibodies covalently bound onto the sensing channel.

Contrary to previous designs, in which two arms (a sensing and a reference one) are separated, a novel configuration based on interferometry but using a single waveguide has been recently developed. The so-called bimodal waveguides (BMWG) [61] is a single waveguide, with two 
different zones: a starting one of single-mode behavior and a second-one which supports two modes (zero and first-order mode) which propagate at different velocities depending on the refractive index of the cladding layer (see Figure 3.E). The interference pattern at the exit of the waveguide changes if the refractive index varies, for instance, as a consequence of a biointeraction event. The configuration is significantly less complex than for conventional interferometers while the sensitivity level achieved is comparable (detection limit of $2.5 \cdot 10^{-7}$ RIU [61]). The sensing chip was fabricated onto a silicon substrate using standard microelectronics technology. $\mathrm{A} \mathrm{Si}_{3} \mathrm{~N}_{4}$ layer $(\mathrm{n}=2.00$, thickness $=350 \mathrm{~nm})$ was deposited onto $\mathrm{SiO}_{2}$ $(\mathrm{n}=1.46$, thickness $=2 \mu \mathrm{m})$ and a ridge type waveguide $(2 \mathrm{~nm}$ height $)$ was formed on top by BHF etching. The single mode region was reduced to $150 \mathrm{~nm}$ thickness. The device is covered with a $\mathrm{SiO}_{2}$ cladding layer. The initial design includes chips with dimensions of $30 \times 10 \mathrm{~mm}^{2}$ and 26 independent interferometers. A preliminary immunosensing of human growth hormone has shown an excellent sensitivity of $30 \mathrm{pg} / \mathrm{mL}$ (Lechuga et al., unpublished results).

\subsection{Grating coupled waveguide sensors}

Grating-coupled waveguide sensors are one of the first transducers earlier developed [62,63]. A grating coupler is a system of periodic disturbance in a planar single-mode waveguide (commonly $\mathrm{SiO}_{2}-\mathrm{TiO}_{2}$ or $\mathrm{Ta}_{2} \mathrm{O}_{5}$ ). The grating allows the excitation of a guided mode of the waveguide at a certain angle of incidence when the incoupling condition is fulfilled:

$$
n_{e f f}=n_{\text {air }} \sin \alpha+l\left(\frac{\lambda}{\Lambda}\right)
$$

Where $n_{\text {eff }}$ is the effective refractive index of the waveguide, $n_{\text {air }}$ is the refractive index of air, $\alpha$ is the angle of incidence of the light, $l$ is the diffraction order, $\lambda$ is the wavelength and $\Lambda$ is the grating period. As can be deduced from equation (4), the incoupling angle is sensitive to any variation in the refractive index at the surface of the waveguide $\left(\mathrm{n}_{\text {eff }}\right)$ within the evanescent field region. Therefore, induced changes in the coupled angle can be used for sensing. Conversely, it is possible to evaluate the outcoupling angle of the guided light.

In the input grating coupler device, s- and p- polarized laser beams are scanned at a variable angle to excite both TE and TM modes. The grating sensor requires a precise mechanical movement of the rotation stage which includes the sensing platform, the fluidics and the photodetectors $[64,65]$. On the other hand, output grating devices are based on the monitoring of the outcoupling angle, which does not require a moving stage, simplifying the setup and decreasing the response time $[66,67]$. The in- and output grating configurations were initially developed by Nellen and Lukosz in the early nineties, showing a refractive index LOD around $10^{-6}$ RIU. Some initial biosensing evaluations using model systems as IgG/antiIgG or 
biotin/streptavidin showed moderate sensitivities. Years later, more in depth biosensing experiments were demonstrated such as competitive immunoassays [68,69], on-line monitoring of monoclonal antibodies production [70], the influence of surface biofunctionalization [71] or the determination of kinetics rate constants [72]. A new setup configuration based on reflectionmode operation avoiding any moving part was developed by Brandenburg et al. [73], which considerably simplified the previous one. The incident polarized light is focused on the grating by a lens in such a way that all the coupling angles in the expected range are included, and the minimum of the reflected light is followed by a CCD camera. A similar bulk LOD ( $3 \cdot 10^{-6}$ RIU) was reached and some biosensing experiments based on competitive immunoassays were reported, as pesticide detection [74], optimization of surface biofunctionalization [49] or DNA hybridization $[75,76]$.

More recently, Grego et al. [77] have used silicon oxynitride $\left(\mathrm{SiO}_{\mathrm{x}} \mathrm{N}_{\mathrm{y}}\right)$ deposited by plasmaenhanced chemical vapor deposition (PECVD) to improve the performance of a grating coupled sensor. Two technologies, colloidal self-assembly and imprint lithography were used in order to incorporate the gratings on the waveguide. With the last one, two dimensional grating structures with optimum pitch in two orthogonal directions were designed and tested in input grating coupler configurations by angular and wavelength interrogation [78]. This 2D structure showed a sensitivity enhancement (a factor of two) as compared with conventional one-dimensional grating sensor. [79]. Yuen and coworkers [80] have developed a self-referencing optical detection system, by using novel microfluidics which efficiently divides the grating in two halves. The grating operates under reflection mode, and the CCD image obtained can be processed to analyze the separate responses.

Wiki et al. [81,82] developed the so-called WIOS (Wavelength Interrogated Optical Sensor), based on grating couplers (Figure 4.A). The approach is based on a single mode waveguide structured with two grating couplers, to in and out-coupling the light, respectively. The change of the refractive index due to an interaction onto the surface is monitored by scanning the resonance peak at a fixed angle of incidence by a tunable laser diode and adjusted with a mirror. The emitted light is collected by a multi-mode fiber and detected by photodiodes. In order to have different in and out coupling angles, two different grating configurations were designed, one with a grating with different periods for in and output and the other one based on a single grating with different thickness layer (see Figure 4.A). Reference pads close to sensing pads are also included for self-referencing, thus improving the signal-to-noise ratio. A compact device including four channels and the laser source, the mirror, the flow cell and other components was also developed and tested for biosensing of low molecular weight analytes, such as biotin, and large biomolecules, such as antibodies, showing mass detection limits of $0.3 \mathrm{pg} / \mathrm{mm}^{2}$. The 
device showed a label-free detection of molecules as small as $200 \mathrm{Da}$. Further development of this integrated device has improved its multiplexed capabilities by using chips with up to 24 different sensing areas (each one with the input and output gratings). Adrian et al. [83] have applied the device with a flow cell of three $7.2 \mu \mathrm{L}$ channels for the detection of sulfonamides, a family of antibiotics widely used in veterinary medicine as feed additives. The test was based on indirect immunoassays where the antigen was covalently immobilized onto the chip surface previously functionalized with a photopolymerizable dextran layer for avoiding non-specific interactions. A limit of detection in milk samples of $0.5 \mu \mathrm{g} / \mathrm{L}$ was achieved but an additional amplification step with a secondary antibody was required. The multiplexed capabilities of the device were tested for the simultaneous detection of four different families of antibiotics [84]. By following a similar protocol as above, four different antigens were spotted in separate sensing areas. The multi-assay, with capability to detect 34 antimicrobials, was done in milk sample showing the same sensitivity with high levels of specificity, indicating the potential of the sensor as a screening analytical tool. The combination of the WIOS configuration with a polymer-based self-contained microfluidic cartridge [85] has added more value to the sensor as a lab-on-a-chip device to be employed at the agroalimentary industry (Figure 4.B). Semiquantitative assays for the simultaneous detection of three different antibiotic families have been developed. Pasche et al. simultaneously detected three different cytokines using a sandwich immunoassay [86] and the sensor was also tested in cell cultures for studying inflammatory processes by the quantification of cytokines released by the cells. In the field of genomics, it has been employed to study the kinetics of covalent immobilization of double-stranded oligonucleotides in order to evaluate the optimal hybridization efficiency after sequential denaturation [87].

\subsection{Ring Resonator sensors}

Ring resonator transducers are increasingly being used for biosensing due to their high sensitivity and their potential to be produced in highly dense arrays for multiplexed analysis. In a ring resonator configuration, light is coupled by an input waveguide via the evanescent field into a circular waveguide. Coupled light propagates through the loop in the form of whispering gallery modes in such a way that constructive interference is generated in the multiple roundtrips over the ring circumference, considerably enhancing the sensitivity. Detection in a ring resonator is based on a refractive index change, which is related to the WGM spectral position through the resonant condition:

$$
\lambda=2 \pi n_{e f f} r / m
$$

where $m$ is an integer describing the WGM angular momentum, $\lambda$ is the wavelength; $r$ is the radius of the ring and $n_{\text {eff }}$ is the effective refractive index experienced by the WGM. A change in 
the effective refractive index of the ring environment due to biointeractions onto the ring surface shifts the resonance spectrum, which can be monitored by scanning the wavelength or by measuring the intensity profile at a fixed wavelength.

Contrary to straight waveguides, in ring resonators interaction is not longer determined by the length of the waveguide but rather by the number of revolutions within the ring, which is indicated by the resonator quality factor ( $\mathrm{Q}$ factor). The effective length $\left(L_{\text {eff }}\right)$ is related to the $\mathrm{Q}$ factor by:

$$
L_{\text {eff }}=Q \lambda / 2 \pi n
$$

Where $\lambda$ is the wavelength and $n$ the refractive index of the ring resonator. High $\mathrm{Q}$ factors indicate low optical losses and long photon lifetimes, which is translated into narrow linewidths and high peak resolution (which means a high sensitivity). Relatively high Q factors of $10^{6}$ can be achieved in resonators of few $\mu \mathrm{m}$ (typically around 50-200 $\mu \mathrm{m}$ ), which is equivalent to have planar waveguides of several $\mathrm{cm}$. For example, a quality factor of $10^{8}$ means that the molecule will be sampled more than 100.000 times [91]. Therefore, despite the small size of the resonator, it could achieve higher sensitivities than straight waveguides while using orders of magnitude less surface area. That is the reason why these devices can be subjected to a high degree of integration.

Few types of integrated optical-based resonating structures have being explored for biosensing, as the planar microring resonators based on microdisks [88-91], microrings [92-97], or microtoroids [98-102]. Compact ring resonators with optimal photonic properties and optimal sensitivity can be fabricated (see Figure 5) including complex configurations incorporating multiple resonators. Different materials such as glass-based [103,104], $\mathrm{Si}_{\mathrm{x}} \mathrm{N}_{\mathrm{y}}-\mathrm{SiO}_{2}$ [105] or polymer-based [106-109] ring resonators have been reported and applied to DNA, proteins or bacteria detection [104] (Figure 5.A.). Recently special attraction is gaining silicon-on-insulator (SOI)-based resonators [110]. De Vos et al. [92] have described SOI microring resonators (5 $\mu \mathrm{m}$ radius, Q factor of 20,000) with a bulk refractive index sensitivity of $70 \mathrm{~nm} / \mathrm{RIU}$, which corresponds to a minimal detectable refractive index shift of $10^{-5}$ RIU. Biosensing evaluation of avidin interaction onto a biotinylated surface led to estimated detection limits around $10 \mathrm{ng} / \mathrm{mL}$ $[92,111]$, which is comparable to other label-free sensors. Recently, the same group has reported an array of SOI microring resonators [112] based on three series of four rings connected to a single input waveguide, one for each series, and each one with individual output waveguides (Figure 5.B.). The incoming and outcoming light is coupled by grating couplers and the collected signal of the twelve resonators is imaged on an infrared camera. The four resonators in 
each series have different circumference ratio (with variations about $30 \mathrm{~nm}$ ) showing independent and not overlapped resonance spectra. A PDMS flow cell with independent microfluidic channels $\left(200 \times 50 \mu \mathrm{m}^{2}\right)$ was mounted on top of the chip for sample delivery. The multiplexed capabilities were evaluated by immobilizing three different proteins and by successively flowing two of the three specific antibodies. Due to the optimized surface biofunctionalization, the device showed high specificity and low non-specific adsorption (which could be also corrected by one resonator acting as reference) [111]. With an estimated peak resolution around $0.4 \mathrm{pm}$ and a $\mathrm{Q}$ factor around $2 \cdot 10^{4}$, a surface mass detection limit resulted in $3.4 \mathrm{pg} / \mathrm{mm}^{2}$.

Li et al. [94] proposed a two concentric ring structure in order to increase the notch depth and the sensing area at the same time. The sensor was fabricated over a SOI wafer with an outer radius of $21 \mu \mathrm{m}$, and an inner radius of $20 \mu \mathrm{m}$, and an air gap of $480 \mathrm{~nm}$ between them, coupled to a waveguide of $10 \mu \mathrm{m}$ width tapered down to $480 \mathrm{~nm}$. The estimated $\mathrm{Q}$ factor of this resonator was around 5.1.10 ${ }^{4}$ and bulk sensitivity was considerably high, of $683 \mathrm{~nm} / \mathrm{RIU}$. Iqbal et al. [96] have recently developed a biosensing platform based on the integration of 32 ring resonators accessed via a bus waveguide, which are simultaneously interrogated (Figure 5.C.). 24 of them are used as sensing resonators, with an etched window on the surface for sample interaction, whereas the reminding eight, left under the cladding layer, are used as reference for temperature-induced drift. Light is coupled in and out by grating couplers. The chip is covered with a flow cell that comprises the fluidic ports, channels, and reservoirs for fluid delivery to the 32 sensors by using gaskets that define the channels in each sensing area. The optical setup is complex but allows simultaneous reading of 24 interactions. A RI detection limit of $7.6 \cdot 10^{-7}$ was achieved [96], with good repeatability among the different resonators and an estimated surface LOD of $1.5 \mathrm{pg} / \mathrm{mm}^{2}$ [97]. AntiIgG/IgG and biotin/streptavidin interactions were used as proofof-concept systems in order to assess the biosensing capabilities, and a preliminary real-time multiplexed analysis of DNA hybridization showed the viability of the device [96]. From the strict biosensing point of view, this platform has been applied few times for in-depth bioanalytical problems. For instance, by immobilizing specific antibodies [113], cytokines have been detected at low concentrations (below $0.1 \mathrm{ng} / \mathrm{mL}$ in buffer) [114] but incorporating a secondary antibody for amplification of the signal. A sandwich assay was applied to the detection of cellular secretion of cytokines, showing the viability of measuring them in cell culture media, while retaining the sensitivity. Moreover, regeneration of the antibody layer was achieved, a step not always contemplated in experiments with biosensors. The use of several microrings $(n=15)$ diminishes the uncertainties, and increases the reliability of the tests. Similarly, it has been detected a cancer biomarker by immobilizing the specific antibodies for carcinoembryonic antigen (CEA) in a single microring [115] achieving a detection limit around 
$25 \mathrm{ng} / \mathrm{mL}$ in a complex media like serum, which is close to relevant diagnostic concentrations. The multiplexed capabilities of the arrayed resonators were evaluated by immobilizing six different antibodies (five specific antibodies and one non-specific as control, four microrings for each one, see Figure 5.C.) for the multiplexed determination of five relevant proteins. Prostate specific antigen (PSA), $\alpha$-fetoprotein (AFP), carcinoembryonic antigen (CEA), $\alpha$-tumor necrosis factor (TNF- $\alpha$ ), and interleukin-8 (IL-8) were simultaneously detected in an unknown protein cocktail solution [116]. A fast (10 min), sensitive $(<150 \mathrm{fmol})$ and simultaneous detection of multiple micro RNAs (miRNAs) using single strand DNA as immobilized probes onto the microring array (four different sequences) has also been recently published [117]. Single nucleotide polymorphism could even be discriminated and expression levels of miRNA in cells were also determined.

Toroidal-shaped based microcavities offer resolution levels significantly higher than previous structures, since they exhibit ultrahigh Q factors $\left(>10^{8}\right)[118,119]$ while preserving the same advantages for mass production and integration capabilities as planar ring resonators. Microtoroids can be fabricated in arrays over silicon wafer using standard lithography techniques (see Figure 5.D.) with dimensions ranging from 30 to $150 \mu \mathrm{m}$ for the major diameter to 2.5-6 $\mu \mathrm{m}$ for the minor diameter. The structures are coupled to a low-loss tapered optical fiber and have been evaluated for biosensing by immobilizing specific antibodies using protein $\mathrm{G}$ for oriented attachment. With this configuration, cytokines were detected at extremely low levels, with a LOD around $5 \mathrm{aM}\left(5 \cdot 10^{-18} \mathrm{M}\right)$, showing a wide working range of around 12 orders of magnitude in buffer conditions and with the remarkable capability of resolving single molecule detection [98]. The capabilities of the sensor were also evaluated in 10-fold diluted serum. The same group has attempted the fabrication of low-loss polymer [99] or polymer-silica hybrid [120] toroids, which, even with a lower $\mathrm{Q}$ factors $\left(10^{5}-10^{7}\right)$, are still significantly competitive with other ring resonators. Nevertheless their biosensing capabilities have not been shown yet.

\subsection{Photonic crystal waveguide sensors}

Photonic crystal-based biosensors are a relatively novel technology which is gaining much attention and is growingly appearing in literature [121,122]. Photonic crystals are well defined nanostructures with periodically repeated variations in the refractive index in one, two or three orthogonal directions generating one, two or three dimensional photonic crystals (1D, 2D or 3D $\mathrm{PhC}$ ). The lattice structure is generated on the length scale of the light wavelength, which generates photonic bandgaps where light cannot propagate in the crystal. The width and position of the photonic gap is highly dependent on the refractive index change between the dielectric materials and on the periodicity of the structure [123], which is exploited for sensing. 
Integrated photonic crystals have been interestingly applied in dielectric mirrors, lasers, narrowband optical filters, microresonators and in waveguide structures and due to their high degree of integration they are ideal candidates for lab-on-chip biosensing. Device structures based on linear gratings and 2- dimensional gratings (i.e. arrays of holes arranged in checkerboard or hexagonal close-packed grids along the sensor surface) are the simplest ones in terms of fabrication and sensing. The introduction of discrete or line defects on the grating structure generates transducer cavities with enhanced sensitivity. The strong confinement of the light in the periodic lattice makes these materials very attractive not only because of the sensitivity but also due to their small dimensions, limited to few $\mu \mathrm{m}^{2}$, which allows for low sample consumption.

Cunningham's Group has pioneered the development of bulk photonic crystal based biosensors. [124-126]. They have implemented a one-dimensional subwavelength polymer grating coated with a layer of high refractive index $\left(\mathrm{TiO}_{2}\right)$ [127]. White light is perpendicularly impinged on the sensor surface and the reflected light is collected, which shifts after a biomolecular interaction takes place. The fabrication has been implemented into continuous sheets of plastic films. The interrogation method can read individually several areas of the surface, avoiding optical crosstalk between adjacent sensor regions. The overall technology offers the possibility to produce low cost, disposable sensing surfaces with high throughput capabilities [128]. In addition, the sensing surfaces have been adapted to be allocated at the bottom of microtiter plates (96, 384 or 1536 formats) [128] or microarray slides [128]. Moreover, they can be integrated within microfluidic networks by replica molding of the photonic crystal sensors and fluid channels in a simultaneous process [129-131]. The latter configuration permits kinetic and high resolution endpoint spatial measurements, improving the detection time and decreasing the detection limit as compared to standard in-well detection [132]. Recently, a microfluidic array chip with combinatorial mixing and on-chip sensing capabilities has also been demonstrated using AtC (Actuate to Close) microfluidic networks [133]. The sensors have been exploited for a wide range of bioassays [134], including cell-based assays, characterization and study of cells attachment to surfaces [135-137], protein and virus detection [132] [138], study of small molecules aggregation for drug screening [139], or for protein-protein interactions [140], among others. The technology based on standard microplate-based assays has been commercialized by SRU Biosystems (www.srubiosystems.com) (see Section 4).

Photonic crystals with a defined structure can offer interesting alternatives. Beside few examples, such as a pillar-based array of 2D photonic crystals [141] recently developed but not evaluated for biosensing, most of the work is focused on hole-array PhC (see Figure 6.A.). 
Planar photonic waveguides, where a guided wave is generated by missing holes or defects in the bulk photonic crystal structure have been fabricated on SOI wafer. Unfortunately, and despite the large effort invested, scarce biosensing examples can be found in the literature and such examples are only at the proof of concept level. Most of the work is mainly focused on fabrication, simulation experiments and bulk refractive index evaluation to establish the sensitivity of the structures.

Some examples are worthy to be mentioned as the work by Dofner et al. who designed and fabricated a SOI photonic crystal waveguide consisting of a hexagonal lattice of holes with a single missing row line defect and point cavities in different arrangements (either a defect based on three missing holes, a single hole or a shifted line of holes arranged perpendicular to the waveguide orientation) (see Figure 6.A. bottom)[142,143]. The optical properties of all the structures were assessed by TE polarized light coupled in and out of the ridge waveguide by a tapered fiber, allowing in-planar-geometry evaluation. By placing a flow cell on top of the structure, refractive index sensing in the most optimal case ( $Q$ factor 3000) gave a bulk LOD of $\sim 10^{-3}$ RIU [142]. Surface sensitivity was assessed by physisorption of BSA or avidin as model proteins, giving a mass sensitivity around $24.7 \mathrm{~nm} / \mathrm{pg}$, with a minimal detectable mass of around $4 \mathrm{fg}$ and a detection limit of $\sim 500 \mathrm{pg} / \mathrm{mm}^{2}$.

Skivesen et al. [144] have fabricated a waveguide with holes of $240 \mathrm{~nm}$ in diameter, a lattice constant of $370 \mathrm{~nm}$ and a length of $25 \mu \mathrm{m}$, using electron-beam lithography and inductively coupled plasma etching. A BSA adsorption was demonstrated under static conditions. A similar structure has been used for protein and DNA sensing $[145,146]$. The photonic crystal length was $20 \mu \mathrm{m}$ with a lattice constant and hole radius of 390 and $111 \mathrm{~nm}$, respectively. A $500 \mathrm{~nm}$ wide single-mode access waveguide was employed to obtain a sharp band edge (see Figure 6.A). A LOD of $6 \cdot 10^{-4}$ RIU was achieved [145]. Tapered fibers were used for the in- and outcoupling of TE polarized light and a flow cell was mounted on top of the sensing surface. Hybridization of DNA strands was monitored in the nM range [146]. For proteins, anti-BSA was detected over a BSA-functionalized surface, reaching a mass detection limit of $2.1 \mathrm{pg} / \mathrm{mm}^{2}$ (which means a total mass detection limit of $0.7 \mathrm{fg}$ ).

1D photonic crystal microcavities fabricated by x-ray lithography on SOI wafer have also been described [147], introducing defects in the structure by breaking the periodicity of the holes. The resonant wavelength can be tuned according to the defect cavity spacing. This type of structure has been adapted by Erickson's group for the development of the so-called Nanoscale Optofluidic Sensor Array (NOSA). This sensor has a microcavity structure (resonator) that is evanescently coupled to an adjacent single-mode silicon waveguide. The design of the structure 
includes parallel resonator arrays with slightly different cavity spacing and, therefore, different resonant wavelength and with individual Q factors between 1000 and 3000, all of them placed along a single waveguide (see Figure 6.B.). Nanotapers were used for light coupling and a PDMS flow channel running orthogonally to the array was mounted onto the chip, for simultaneous and multiplexed detection [148]. Bulk refractive index LOD of $7 \cdot 10^{-5}$ RIU was experimentally determined, which could be translated into a high sensitivity for mass detection, when considering the extremely high confinement in the inner holes. Detection of serotypes of Dengue virus was demonstrated by immobilizing four different DNA probes in different resonators and subsequently adding the specific DNA target for one of them [149]. These experiments only showed a proof-of-concept for nucleic acid interaction. Similarly, immunochemical multiplexed detection of interleukins (IL) was also addressed [150]. Three different specific antibodies for IL4, 6, and 8, respectively, were immobilized and a sandwich assay was performed by adding the target followed by a secondary specific antibody in order to enhance the signal. It is worth noticing that the device has low sensitivity since it has moderate Q factors (3000), which may be enhanced by optimizing the crystal structure (such as the gap and hole dimensions). Although the sensitivity of this $\mathrm{PhC}$ sensor is not high, this is one of the few examples showing biosensing capabilities while including a multiplexed configuration.

Fauchet el $a l$. have also made a strong contribution in this area by developing two dimensional photonic crystal-based structures [151]. Biotin-streptavidin interaction and covalent immobilization of BSA were evaluated and a minimum mass coverage of around $2.5 \mathrm{fg}$ was detected [151]. SOI-based wafers were used to fabricate photonic crystal waveguides with a defect line in the structure to guide the light. Recently, a structure where the defect line is generated adjacent to the photonic microcavities has been proposed in such a way that the device can operate as a multi-channel sensor [152,153]. This structure shows a limited sensitivity with Q factors around 400 and bulk LOD in the range of $10^{-2}$ RIU. Biosensing evaluation was done under static regime, with incubation steps and evaluation in dry environment. The surface was covalently modified with antibodies (anti-IgG) and target antibodies (IgG) were detected, observing a detection limit around $67 \mathrm{nM}(10 \mu \mathrm{g} / \mathrm{mL})$ which corresponds to a surface sensitivity of $2.3 \cdot 10^{5} \mathrm{~nm} / \mathrm{M}$ [153]. Considering that the major contribution to the signal comes from the most sensitive area of the region (the defect in the structure and the surrounding holes) a surface density of $1 \mathrm{ng} / \mathrm{mm}^{2}$ and a minimum mass of 1.5 fg could be detected.

Zlatanovic et al. designed a photonic crystal (Figure 6.C) structure with a ridge waveguide but with low Q factors (around 400) [154]. Real time biosensing was demonstrated by immobilizing biotin-BSA and detecting specific antibodies against biotin. An excellent limit of detection of 
$20 \mathrm{pM}$ of antibody was achieved, which in terms of mass and considering the affinity of the pair antibody/antigen was found to be around $4.5 \mathrm{fg}$. Moreover the experiments included the regeneration of the bioactive surface. These results demonstrated that despite the low resolution of the device (due to the low $\mathrm{Q}$ factor), an adequate biofunctionalization of the surface could lead to acceptable sensitivities for biodetection.

Overall, photonic crystal structures still remain as a promising type of transducers with moderate sensitivities as compared with other label free photonic sensors. Better features could be achieved by optimizing the crystal and defect dimensions and also by positioning the bioreceptor only in the holes (by controlled immobilization), where the confinement is maximum, therefore resulting in highest resonant shifts. In fact, a growing number of examples are appearing in the literature focused on single particle detection by trapping them at the holes; single virus or small pathogens could be detected in this way [155-157].

\subsection{Silicon wires, slot waveguides and other nanophotonic sensing configurations}

In this section we include an overview of the new and emerging trends in the design and implementation of nanophotonic structures for sensing, which we envisage could be highly competitive with the ones described in previous sections, either due to their sensitivity or to their capabilities towards integration and miniaturization. Among them, slot waveguide-based structures and silicon wires stand out for their potential enhanced sensitivity due to the confinement of the electromagnetic field and for their highly integration capabilities in compact dense arrays of individual sensors with versatile geometries. Unfortunately, few biosensing experiments have been demonstrated with these devices, and only proof-of-concept experiments with model systems have been published so far.

Silicon photonic wires are submicron channel waveguides fabricated by electron beam lithography and reactive ion etching on SOI wafers. The high index contrast between the silicon core $(n=3.5)$ and silica cladding $(n=1.5)$ yields a strong field confinement and allows for sharp waveguide bends radii of few microns with low losses. With this technology the fabrication of waveguides with higher compactness and higher intrinsic sensitivity (provided by the TM mode) than the lower-contrast index material counterparts is possible . Initially developed for optical switches, directional couplers and other telecommunications applications, silicon wires first showed up in the biosensing field by the pioneer work of Janz's group. This group first described a Mach-Zehnder interferometer based on SOI photonic wire waveguides [158] and a double spiral millimeter-long resonator folded within a compact size of 100-150 $\mu \mathrm{m}$ (and a Q factor of 17600) (Figure 7.A.). Both sensors were fabricated on SOI wafer with a $\mathrm{Si}$ core thickness of $0.26 \mu \mathrm{m}$ and a buried $\mathrm{SiO}_{2}$ cladding of $2 \mu \mathrm{m}$. Single mode wire waveguides of 450 
$\mathrm{nm}$ width were selected and a SU-8 cover layer was employed to isolate the non-sensing regions. Bulk sensitivity and surface biomolecular interactions were evaluated under flow regime (using a PDMS microflow cell). Updated versions incorporate improvements such as a reference arm to suppress wavelength and temperature drift $[159,160]$, integration of dense arrays of six spiral MZI with an SU-8 fluidic channel for the delivery of liquids [161], or the use of four resonators [162], which overall opens up the possibility to perform multiplexed analyses (see Figure 7.A.). The dimensions of the folded wires are suitable for a controlled surface functionalization using conventional spotters. The performance of the sensing devices was assessed by immobilizing two different antibodies in individual sensors and simultaneously evaluating them with the corresponding anti-IgG. For the MZI configuration, TM polarized light is delivered by an optical fiber which splits the light to the six sensors; parallel readout of the light exiting the six output waveguides is done by a near infrared camera. Results have shown the discrimination between the different biomodified surfaces, demonstrating the high potential of silicon wires-based devices for multiplexed biosensing. However, results are not yet conclusive about the sensitivity of these sensors and only estimated values of resolvable surface coverage around $0.25 \mathrm{pg} / \mathrm{mm}^{2}$ are theoretically predicted (corresponding to a detected molecular mass of $0.5 \mathrm{fg}$ ) [161]. In the case of resonators, with radius between 20 and $28 \mu \mathrm{m}$ (and improved Q factors of 25000) and a reference included in the structure, a bulk sensitivity of 135 $\mathrm{nm} / \mathrm{RIU}\left(<2 \cdot 10^{-6} \mathrm{RIU}\right)$ has been achieved and a surface immunochemical detection down to 20 $\mathrm{pM}$ (resolvable mass of $40 \mathrm{ag}$ ) [162] has been shown so far.

Slot-waveguides [163-165] consist of two slabs of high refractive index materials separated by a nanometer-scale low refractive index slot region and surrounded by low-refractive index cladding materials in such a way that light is strongly confined in the slot region. A stronger light-analyte interaction can be achieved within this region as compared to conventional rib or planar waveguides, which results in an enhanced sensitivity. Moreover, slot-waveguides can be fabricated within structures such as resonators or interferometers by employing CMOS compatible materials and technology, including SOI technology, which enables miniaturization, and further integration within lab-on-a-chip platforms.

So far, slot-waveguide resonators have been reported by only two research groups. Barrios et al. pioneered the slot waveguide configuration, using $\mathrm{Si}_{3} \mathrm{O}_{4}-\mathrm{SiO}_{2}$ for the fabrication of a slotwaveguide ring resonator structure [166], with a radius of $70 \mu \mathrm{m}$ and slots of $200 \mathrm{~nm}$ for both the waveguide and the resonator. A bulk sensitivity of $212 \mathrm{~nm} / \mathrm{RIU}$ for a resonator with a $\mathrm{Q}$ factor of 1800 was reached, significantly better than conventional resonators, indicating a considerably contribution from the slot region. Biosensing evaluation was done off-flow by covalently immobilizing antibodies onto the surface and detecting their specific target (in this 
case BSA as model system) showing a surface limit of detection of $16 \mathrm{pg} / \mathrm{mm}^{2}$ [167], which indicates a poor to moderate sensitivity as compared to most of the optical biosensors (see Table 1 for comparison). Recently, a highly integrated chip based on an array of eight slot-waveguide ring resonators (two references-one for laser alignment and compensation and another one for control- and six for parallel analyses) have been fabricated [168], including the microfluidics integrated with the sensor in a cartridge $(40 \mathrm{~mm} \times 15 \mathrm{~mm})$ and the alignment with the read-out instrumentation for simultaneous detection (see Figure 7.B). The incoming light is coupled by fully etched surface grating couplers, and split by multimode interference splitters to all the channels (reference and sensing ones included). The outcoupling is done by imaging the waveguide end faces onto a photodiode array. Each sensing area is individually reached using a single PDMS flow cell. Bulk detection limit was $5 \times 10^{-6}$ RIU, similar to the one previously reported for individual sensors, which is considerably good as compared to conventional resonators or single slot sensing configuration. A surface mass detection limit of $0.9 \mathrm{pg} / \mathrm{mm}^{2}$ was achieved, which is significantly better than previous values, mainly due to the use of reference channels for compensation of temperature drifts, and to the use of a laser with smaller wavelength step [169]. Claes et al.[95] adapted their work with conventional resonators to the development of a SOI-based slot waveguide ring resonator. They simulated the optimum dimensions of the photonic structure (slot and rib width) in order to obtain the maximum resonance shift to make it feasible for biosensing. A ring resonator ( $5 \mu \mathrm{m}$ bending radius) with a $104 \mathrm{~nm}$ slot width, $268 \mathrm{~nm}$ rib and $220 \mathrm{~nm}$ height, with two identical bus waveguides was fabricated to get the maximum light coupling via single-mode fibers vertically coupled with gratings. With this resonator, a four times better bulk sensitivity ( 298 vs $70 \mathrm{~nm} / \mathrm{RIU}$ ) was reached as compared with conventional waveguide resonator.

Instead of ring structure with a vertical slot, Lee et al. $[89,90]$ designed a horizontal air-slotted silicon nitride microdisk resonator. In particular, $15 \mu \mathrm{m}$ diameter microdisks were fabricated by a multilayer deposition $\left(240 \mathrm{~nm} \mathrm{SiN} / 40 \mathrm{~nm} \mathrm{SiO} / 240 \mathrm{~nm} \mathrm{SiN}_{\mathrm{x}}\right.$ ) on a silicon surface and selective wet etching. A further hydrofluoric acid etch of the $\mathrm{SiO}_{2}$ defines a $2 \mu \mathrm{m}$ deep horizontal air slot from the disk edge with a thickness of $40 \mathrm{~nm}$. In this way, it is possible to obtain ultra-thin slots with high smoothness in the slot walls (see Figure 7.C) and an enhancement of the sensitivity due to the spatial confinement in the slot region. Microdisks with Q factors of $~ 7000$ were obtained. By using a $1 \mu \mathrm{m}$ tapered fiber to couple the light (TM mode) in and out, surface sensitivity was evaluated by assessing the resonance shift of the fundamental radial mode at various streptavidin concentrations added onto a disk previously modified with biotin groups. A value of $2.5 \mathrm{~nm} /(\mu \mathrm{g} / \mathrm{mL})$ was obtained; considering a system resolution of 78 $\mathrm{pm}$, a limit of detection of $30 \mathrm{ng} / \mathrm{mL}$ can be extrapolated, which indicates a moderate 
sensitivity. Nevertheless, measurements with microdisks lacking the horizontal slot would allow extracting the contribution of the slot region in the sensitivity.

Recently Di Falco have combined slotted waveguides with a planar photonic crystal platform [170], resulting in cavities with extremely high sensitivity as compared with conventional waveguide photonic crystals, reaching Q factors up to 50000 in air (4000 in liquid), a sensitivity of $1500 \mathrm{~nm} / \mathrm{RIU}$, and a detection limit around $7 \cdot 10^{-6}$ RIU, quite remarkable for photonic crystals, as discussed in the section above [171]. Unfortunately, no biosensing experiments with these structures have been performed yet.

Overall, for slot nanophotonic structures, where the enhancement of sensitivity comes precisely from the slot area, a key aspect to address is the controlled and optimum biofunctionalization only in the slot region. This becomes especially tricky since the dimensions of the slot, usually around 100-200 nm, can hinder the complete filling of the area when sample and solutions are delivered. It is of paramount importance to design optimal micro or nanofluidics, which, in combination with proper surface modification, ensures a complete and uniform delivery of the solution and an appropriate wettability of the surface. Until solving the current technical limitations, slot waveguide sensors will not be able to show the exceptional performances for biosensing theoretically predicted.

An original type of integrated photonic structures has recently been developed by Holgado et $a l$., with significant novelties in terms of read-out interrogation at micron and sub-micron sensing spot size. The group has designed a lattice of submicron photonic nanostructures of $\mathrm{SiO}_{2} / \mathrm{Si}$ or polymer pillars whose fabrication is compatible with standard semiconductor technology at wafer level (see figure 7.D). Discrete photonic cells based on (i) cone-shaped holes of $650 \mathrm{~nm}$ top diameter and 396 bottom diameter and lattice parameter of $800 \mathrm{~nm}$ [172] and (ii) SU-8 nanopillars of $499 \mathrm{~nm}$ height, $136 \mathrm{~nm}$ width and $750 \mathrm{~nm}$ lattice pitch [173] have been fabricated. An optical vertical interrogation at the single nanostructure level has been implemented based on three independent reflectivity measurements (as a function of wavelength by spectrometry, as a function of incidence angle by reflectometry, and as a function of phase shift by ellipsometry) $[172,174]$. Reflectivity measurements are very sensitive to any change of the refractive index in close contact to the pillars which can be used for the development of highly sensitive biosensors after an appropriate biofunctionalization.

This novel label-free biosensing scheme has several advantages, besides those derived from the microelectronics-based fabrication: (i) the use of three different readout techniques improves the reproducibility and the reliability of the measurements; (ii) depending on the dimensions of the 
nanostructures and the separation among them, the evanescent field can be strongly confined in those areas, increasing the surface sensitivity towards biomolecule interactions; (iii) as the optical sensing is based on the use of a tightly vertically focused beam, light coupling is avoided, which simplifies the analyses and enables evaluation at spots of $0.9 \mu \mathrm{m}$. As the dimensions of individual structures are submicron-sized, it is possible to analyze single structures. Overall, all these aspects are very attractive for future commercialization of integrated and disposable chips with high throughput capabilities.

Besides the characterization of the structure using solutions with different refractive indexes [172], preliminary biosensing experiments have recently been performed by covalent immobilization of BSA onto the nanopillars and the subsequent biodetection of the interaction with the specific antibody, reaching sensitivities around $3 \mathrm{ng} / \mathrm{mL}$ [175] under static conditions. Further implementation of an appropriate microfluidic flow cell and a flow delivery system will allow the optimum infiltration of the solutions through the whole sensor lattice. This will be the next step for these promising devices that could offer multiplexing evaluation of at least 1200 samples per hour.

\subsection{Optonanomechanical sensors}

Optomechanical (OM) sensing is a novel technology derived from the standard micro and nanoelectromechanical systems widely employed during the last years in the biosensing field. Specifically, nanomechanical systems based on silicon microcantilevers have demonstrated an extremely high sensitivity in the measurement of frequency [176,177] and deflection changes [178] due to mass accumulation or biosensing interactions [179-183], and in the measurement of forces between single molecules [184] or even of forces arising from single electron spin [185].

However, in order to achieve full working prototypes as lab-on-a-chip biosensors, the commonly used optical readout method for nanomechanical sensors have severe limitations related to the complex optical alignment of multiple cantilevers at the same time, and the diffraction constraints when the size of the cantilever is reduced below the wavelength. In an optonanomechanical sensor, the cantilever itself is an optical waveguide which output intensity is a function of the bending induced by a biomolecular interaction. With this new configuration is possible to overcome the aforementioned limitations of standard nanomechanical sensors. This microscale optical waveguide cantilevers are mainly addressed to sensing applications and several works have demonstrated its motion sensitivity. The working principle of the optical microcantilevers do not rely on refractive index changes, but on the microcantilever bending or resonance frequency shift, measured through the light intensity collected at the cantilever free end. The limit of detection can be evaluated in terms of analyte concentration (i.e. in $\mathrm{ng} / \mathrm{mL}$ or 
molarity), according to the cantilever bending (in $\mathrm{nm}$ ) or frequency change (Hz), or according to surface mass density $\left(\mathrm{pg} / \mathrm{mm}^{2}\right)$.

The most extended configuration for biosensing is the end-coupled which consists of a microcantilever acting as a waveguide; the light propagates along the cantilever until reaching the free end. After crossing a small gap, light is collected by an output waveguide (see Figure 8.A). Several parameters can have an influence on the system sensitivity, such as the distance from the cantilever edge to the collecting waveguide, the coupling efficiency or the cantilever material and rigidity. Different materials, cantilever shapes, and light coupling methods have been investigated. The device fabricated by Zinoviev et al. employs an array of 20 waveguide silicon dioxide microcantilevers, each one of them connected with a silicon nitride multimode input and output waveguide; the light from the input waveguide is coupled to the cantilever through the overlapping of the evanescent field [186] (Figure 8.B). With this configuration, the cantilever displacement can be detected with a resolution of $18 \mathrm{fm} \mathrm{Hz}^{-1 / 2}$, sensitivity comparable to the standard optical lever method, which indicates its suitability for biosensing applications.

A silicon microcantilever etched to form a single mode waveguide rib, with a capture asymmetrical multimode waveguide that terminates in a Y-branch, was proposed by Noh et al. [187]. The Y-branch forms a differential signal that is monotonically dependent on the microcantilever deflection, achieving a signal sensitivity of $1.4 \times 10^{-4} \mathrm{~nm}^{-1}$. More recently, the same group has reported a sensitivity enhancement by modifying the mode structure of doublestep rib waveguides used to capture light, reaching a sensitivity comparable to the optical lever method [188]. Besides the use of silicon material, there is an increasing interest on polymeric materials for waveguides fabrication. Employing SU-8 [189-192] and PDMS [193] polymers several groups have already developed integrated optical waveguide cantilevers. Indium phosphide has also been employed showing up as a suitable material for the fabrication of electrostatically actuated end-coupled optical waveguide MEMs [194]. Other works suggest the use of silicon photonic crystals for guiding the light through the microcantilever [195-197], or on chip in-plane Fabry-Pérot interferometer readout [198]. Working with arrays of optical waveguide microcantilevers is more challenging as a multiplexed input of the light in each cantilever must be achieved. With this aim, different strategies have been proposed, such as using diffraction gratings [199], a network of waveguide splitters [200] or integrated silicon light sources and detectors self-aligned to silicon waveguides [201]. A higher integration and scale reduction of these nanophotonic platforms allow the direct actuation of MEMs by the force exerted by the propagated photons. Li et al. suggested two different integrated photonic platforms designs [202,203]: an embedded beam resonator with an on-chip interferometer for the displacement sensing, and a pair end-to-end coupled waveguide cantilevers. Both platforms 
include two input/output grating couplers at the end of the waveguides for light coupling. A displacement sensitivity of $40 \mathrm{fm} \mathrm{Hz}^{-1 / 2}$ was achieved with the end-to-end coupled cantilevers working under vacuum conditions.

However, to our knowledge, the application of this technology to the biosensor field is scarce. For example, Koev et al. were able to detect homocysteine in liquid at a lowest concentration of $10 \mu \mathrm{M}$ with a minimum detectable cantilever bending of $5 \mathrm{~nm}$ [191]. Nordin's group has recently demonstrated the integration of a 16 microcantilever array using in-plane photonic readout with a 2-layer PDMS-based microfluidic chip which integrated valves to control the fluid flow through the microchannels. However, only the study of the transient response of the microcantilevers was reported. [204] A higher integration and scale reduction of these mechanophotonic platforms can be easily achieved, producing devices with a very small mass and low dissipation, ideal for fundamental studies [205-208], although with no biological applications reported so far.

\section{Current commercial technologies based on label-free photonic biosensors}

Some of the technologies described in the previous sections have evolved into commercial platforms for label-free detection, which has exponentially expanded the number and variety of applications in biotechnology, biology, and clinical analysis. In this section we will give an overview of the most relevant biosensor devices based on integrated optical transducers that have been successfully transferred into the market. As it can be appreciated, none of them has achieved the status of complete "lab-on-a-chip" portable platform with compact and reduced size. Instead, most of them are bulky instruments mainly for laboratory use with a complex and challenging road to achieve point-of-care status. However, all of them have demonstrated the excellent performances of the integrated optics-based sensors in terms of sensitivity and automation, and, in some cases, even in terms of multiplexing (allowing medium to high throughput analysis).

\section{Farfield}

In 2000 Farfield Group launched into the market the AnaLight ${ }^{\circledR}$ interferometer based on Dual Polarization Interferometry (DPI). Whereas all the interferometric schemes previously discussed employ only one polarization, DPI uses both TM and TE polarizations in a sensing transducer that mimics Young configuration. By simultaneously measuring both polarizations, determination of the thickness and the refractive index of a film adsorbed on the sensor surface can be done under real time conditions, which increases the versatility of the device for advanced and complex measurements. Sensitivity of the instrument reaches refractive index variation of $10^{-7} \mathrm{RIU}$, and a mass surface sensitivity of $0.1 \mathrm{pg} / \mathrm{mm}^{2}$. Two simultaneous 
evaluations are possible by a dual-channel flow cell with small sample-volume capability $(<50$ $\mu \mathrm{L}$ ). A bulkier platform, AnaLight ${ }^{\circledR} 4 \mathrm{D}$ Workstation (Analysis Station: 90x48x35 cm and Pump Size $28 \times 23 \times 16 \mathrm{~cm}$ ) is also commercialized for automatic sampling using vials, and 96 and 384 wells format. The instrument has been exploited for diverse biosensing evaluations, using surfaces already preactivated and functionalized with appropriate chemistry. Among others, the platform allows for thermodynamics, kinetics and affinity studies, such as protein interaction studies, protein conformational changes, specific biodetection and biomolecular interactions, surface density evaluation, lipid bilayer formation and characterization, biocompatibility studies and protein or lipid aggregation analyses. The main disadvantage is the limitation to only two samples due to the microfluidics system and the read-out electronics.

\section{SRU Biosystems}

SRU Biosystems, Inc. has developed the BINDTM Biosensor, based on the use of photonic crystals in a grating configuration. The platform offers high multiplexed capabilities in a microplate format (up to 1536 wells). The instrument has a high level of resolution (in the pm level) and a broad dynamic range, which ensures the analysis of both small molecules and large entities as cells or bacteria. The sensing surfaces are modified following optimized protocols to enable both cell-based and biochemical assays with excellent performance. Different readers are available both for single point mode and imaging mode. The instrument has fast detection times (96-well in 30 seconds, 384-well in 1 minute, 1536-well in 3 minutes), or even faster with an advanced readout mode (96-well in 8 seconds, 384-well in 15 seconds, 1536-well in 45 seconds). Among others, the platform has demonstrated its usefulness in diverse applications as for cell morphology and adhesion assays, ion channel assays, small molecule and fragment screening, and for the analysis of interactions of large genomic, protein, peptide or antibody libraries against a wide range of biochemical targets.

\section{Corning}

A quite similar technology to BIND ${ }^{\mathrm{TM}}$ Biosensor has been marketed by Corning. Epic ${ }^{\circledR}$ System is a high throughput screening platform based on waveguide resonant gratings. This device reaches a detection limit around $5 \mathrm{pg} / \mathrm{mm}^{2}$ and has a broad dynamic range. Some functionalized surfaces are available (three types: cell-based, biochemical or fibronectin-coated, also for cell assays). The instrument can be coupled to an automatic liquid handler in order to enhance its throughput performance and has a rate analysis of $6 \mathrm{~s} /$ microplate requiring sample volumes of 15-80 $\mu \mathrm{L}$. A main disadvantage is related to the dimensions of the whole instrumentation $(0.83 \mathrm{~m} \times 1.15 \mathrm{~m} \times 1.98 \mathrm{~m})$ and its high price, which can limit its implementation in laboratories and other institutions. A benchtop version of the reader (EnSpire ${ }^{\circledR}$ Multimode Plate Reader, in collaboration with Perkin-Elmer) is also currently commercialized, with more convenient 
features, which allows for label-free as well as for fluorescence, absorbance and luminescence detection.

\section{Axela}

Axela group has launched the $\operatorname{dotLab}{ }^{\circledR}$ System, an instrument based on diffractive optical gratings. The grating is fabricated on the lower area of a prism-shaped surface, which is integrated in a $10 \mu \mathrm{L}$ flow channel disposable plastic chip. Each dot lab sensor contains eight assay spots aligned with the linear flow channel for multiple assays. The fluidic controller can work under static, continuous flow or constant mixing mode. The samples and solutions are delivered to the sensor by an automated sampling system (from reagent bottles or 96-well microplates), allowing real time evaluation. The $\operatorname{dot} L a b \AA$ is provided with several attachment chemistries (i.e. streptavidin modified, Protein G, Goat-Anti-Mouse-Fc or for covalent binding) which increases the flexibility for the coupling of bioreceptors to the surface. Features of the device include label-free and quantitative evaluation within a broad dynamic range. The dimensions are comparatively smaller than for SRU and Corning instruments $(43.5 \mathrm{~cm} \mathrm{H}$ x 57,9 $\mathrm{cm} \mathrm{W} \times 69.3 \mathrm{~cm} \mathrm{D}$ ) but the multiplexed capabilities are more limited, since the analysis time is slower and only eight simultaneous tests are possible. The instrument can be used for proteinprotein interaction studies, epitope mapping, or for the detection and quantification of proteins, among others.

\section{Microvacuum}

Microvacuum Ltd. manufactures the OWLS System based on Optical Waveguide Lightmode Spectroscopy [63]. In this sensor, polarized light is coupled by an input grating and guided by total internal reflection to the end of the waveguide where is detected by the photodiodes. The light mode spectrum can be obtained by varying the angle of incidence, whereas the flow cell is fixed over the grating coupler waveguide sensor chip. From the mode spectra it is possible to determine the physical parameters of the cover medium (thickness, refractive index, density etc.). The chip is mounted on a precision goniometer which provides the adjustment of the angle of incidence. It operates under continuous flow rate for real time evaluation. The instrument has a compact small-to-medium size and includes the optomechanical system, the electronics, temperature control and the sensor holder, together with the injection unit (with several volume sample loops), although the fluidic pump is external. The data acquisition and the software evaluate the refractive index and thickness of the adsorbed layer, and provide qualitative kinetics information. The instrument can be applied for biomolecular interaction studies (protein-protein, nucleic acids, membrane protein-lipid bilayer...), cell-based assays, surface coverage characterization, drug screening or kinetic analysis. The sensor surface has general features which can be modified on request to improve performance (i.e. dimensions of the chip, 
dielectric coatings of the surface, passivation or functionalization with silanes). The instrument can be combined with two additional units: an electro-chemical system and a fluorescent measuring system. Unfortunately, the instrument has very limited throughput capabilities since it can only perform single measurements on relatively large chips (around $12 \mathrm{~mm}$ x $8 \mathrm{~mm}$ ).

\section{Genalyte}

Relatively new, Genalyte has started commercializing the Maverick Detection System based on an array of microring resonator silicon sensors. The instrument currently in the market is the third generation and has a compact design, which delivers the samples (typically less than 25 $\mu \mathrm{L})$ from a 96-well plate into the plastic chip consumable which contains the sensing surface, consisting of an array of silicon microrings (from 1 up to 128 in each surface, including 8 controls). The system can perform simultaneous measurements in real time, and results are available in a time scale between 5-45 min, depending on the concentration to be detected. Some of the features of the instrument are the possibility to perform kinetics measurements (using 2 flow channels which delivery samples to 16 sensors each one) or end-point measurements using 96-well footprint; it has a wide dynamic range up to eight orders of magnitude, with sensitivities close to $0.1 \mathrm{ng} / \mathrm{mL}$, a limit of detection around $0.1 \mathrm{fg}$ and it is able to measure targets of molecular weight of only $10 \mathrm{Da}$.

\section{Integration in lab-on-a-chip platforms}

The major challenge in the silicon photonic biosensor field is to achieve fully operative lab-ona-chip platform with on-chip detection. Further efforts are addressed to keep high level of sensitivities and to develop complete Si CMOS compatible integrated devices that incorporate the photonic systems with microfluidics and the optoelectronics [46,209,210]. For the development of a complete photonic lab-on-a-chip device, several units must be incorporated on the same platform: (i) the photonic sensors, (ii) the flow cells and the flow delivery system, including miniaturized valves and pumps (iii) the light sources and photodetectors array or miniaturized CCD cameras (iv) processing electronics (i.e. CMOS) and, (v) final packaging with the required firmware and software (see Figure 9). Integration could be monolithic (all functionalities are incorporated in one single chip) or hybrid (functionalities are separated on several chips). In the monolithic approach photodiodes and lasers can be embedded in the waveguides. Due to economic and technical reasons the hybrid approach is mostly preferred, where the components are settled apart. Even if the individual components are well-known, the subsystem interfaces between them are difficult to optimize and these are still the major barriers to be surpassed. With the hybrid arrangement, the optoelectronics and the read-out system can be reusable and only the cartridge containing the sensing chip with the microfludics for independent applications can be interchanged. 
Most of the work done so far mainly includes the integration of the sensors and the fluidics (which has emerged as a new discipline called "optofluidics") and the development of discrete components for future integration [211] Few examples can be found in the literature of complete lab-on-a-chip developments. For instance Jokerst et al. have developed an integrated evanescent-field multimode Mach-Zehnder interferometric sensor fully compatible with SiCMOS process [212] with a LOD of $2.5 \times 10^{-6}$ RIU. In further refinements a fully integrated version was in place with interferometric sensors of only $5 \mathrm{~mm}$ length which reached a

refractive index sensitivity of $\sim 9.2 \times 10^{-7}$ RIU [212]. Unfortunately, this device has not been

tested yet for biosensing applications.

A monolithically integrated interferometric lab-on-a-chip biosensor is under development within the frame of an European project [213]. The principle of operation of the PYTHIA labon-a-chip is based on broad-Band Mach-Zehnder interferometer sensors and including lasers, photodetectors and microfluidics. Femtosecond laser micromachining is nowadays an emerging tool for the fabrication of monolithic optofluidic devices, allowing the direct fabrication of integrated optical devices and microfluidics in fused silica chips. [32,214]

Lab-on-a-chip with the on-chip detection is still very much a work in progress, but last advances in integrated optics-based biosensors and optofluidics indicate that they could eventually be achieved in the near-medium future. Cutting edge technological progress will give raise to a robust product that could run on an automated system minimizing operator skills.

\section{6- Future outlook}

During last years a myriad of different developments at the biosensor field using many diverse technologies and devices has been appearing, such as nanomechanical or MEMS resonators sensors, semiconductor wires-based transducers, highly integrated microelectrodes, carbon nanotubes-based sensors, localized surface plasmon resonance or SERS in metal nanostructures, but none of them has shown the excellent performances for label-free sensing achieved by photonic and nanophotonic devices based on integrated optics, as can be deduced from their excellent limits of detection in real biosensing (shown along this review). Some commercial optical biosensors have been introduced into the market but most of them are bulky and expensive and only offer a general technology, with a limited number of dedicated applications. 
Integrated optics technology is opening the way towards truly portable lab-on-a-chip platforms which could be employed outside the laboratory. But still limitations in the technology, problems in the integration of all the components in one single microsystem and the connection with real world applications should be surpass. In the near future, we will employ lab-on-a-chip biosensor devices for assessing the safety of our food or beverages, for fast environmental pollution identification and for the fast identification of illness at urgency or by diagnostics people at the doctor office in a more comfortable way than it is done today. Due to the intensive research effort which is being done at public institutions and at small, medium and large private companies there are no doubts that lab-on-chip hand-held devices will be a reality in our future society and will impact very positively our lifestyle.

\section{Acknowledgements}

Authors acknowledge the financial support from Fundación M. Botín and SENA project (TSI020301-2008-11) from the Spanish Ministry of Industry. M.A. acknowledges financial support from Juan de la Cierva Program (MICINN-JDC) from the Spanish Ministry of Science and Innovation. 
References

[1] L. M. Lechuga, in Biosensors and Modern Biospecific Analytical Techniques, edited by L. Gorton ( Elsevier Science BV, Amsterdam, Netherlands, 2005), Vol. XLIV.

[2] J. V. Jokerst, J. W. Jacobson, B. D. Bhagwandin, P. N. Floriano, N. Christodoulides, and J. T. McDevitt, Anal. Chem. 82 (5), 1571 (2010).

[3] F. S. Ligler, Anal. Chem. 81 (2), 519 (2008).

[4] J. Homola, Chem. Rev. 108 (2), 462 (2008).

[5] E. Mauriz, A. Calle, J. J. Manclus, A. Montoya, A. Hildebrandt, D. Barcelo, and L. M. Lechuga, Biosens. Bioelectron. 22 (7), 1410 (2007).

[6] J. Treviño, A. Calle, J. M. RodrÌguez-Frade, M. Mellado, and L. M. Lechuga, Clin. Chim. Acta 403 (1-2), 56 (2009).

[7] L. Carrascosa, A. Calle, and L. Lechuga, Anal. Bioanal. Chem. 393 (4), 1173 (2009).

[8] D. Erickson, S. Mandal, A. Yang, and B. Cordovez, Microfluid. Nanofluid. 4 (1), 33 (2008).

[9] A. L. Washburn and R. C. Bailey, Analyst 136 (2), 227 (2011).

[10] F. Vollmer and S. Arnold, Nat. Meth. 5 (7), 591 (2008).

[11] X. D. Fan, I. M. White, S. I. Shopova, H. Zhu, J. D. Suter, and Y. Sun, Anal. Chim. Acta 620, 8 (2008).

[12] P. Lambeck, V., Meas. Sci. Technol. 17 (8), R93 (2006).

[13] C. Monat, P. Domachuk, and B. J. Eggleton, Nat. Photonics 1 (2), 106 (2007).

[14] L. S. Wong, F. Khan, and J. Micklefield, Chem. Rev. 109 (9), 4025 (2009).

[15] B. Prieto-Simon, M. Campas, and J. L. Marty, Protein Pept. Lett. 15 (8), 757 (2008).

[16] F. Rusmini, Z. Zhong, and J. Feijen, Biomacromolecules 8 (6), 1775 (2007).

[17] J. Diao, D. Ren, J. R. Engstrom, and K. H. Lee, Anal. Biochem. 343 (2), 322 (2005).

[18] A. Arafat, M. Giesbers, M. Rosso, E. J. R. Sudhölter, K. Schroën, R. G. White, L. Yang, M. R. Linford, and H. Zuilhof, Langmuir 23 (11), 6233 (2007).

[19] A. Arafat, K. Schroën, L. C. P. M. de Smet, E. J. R. Sudhölter, and H. Zuilhof, J. Am. Chem. Soc. 126 (28), 8600 (2004).

[20] M.-J. Bañuls, V. González-Pedro, C. A. Barrios, R. Puchades, and Á. Maquieira, Biosens. Bioelectron. 25 (6), 1460 (2010).

[21] P. Wu, P. Hogrebe, and D. W. Grainger, Biosens. Bioelectron. 21 (7), 1252 (2006).

[22] A. Akkoyun and U. Bilitewski, Biosens. Bioelectron. 17 (8), 655 (2002).

[23] S. Sharma, R. W. Johnson, and T. A. Desai, Biosens. Bioelectron. 20 (2), 227 (2004).

[24] M. Cerruti, S. Fissolo, C. Carraro, C. Ricciardi, A. Majumdar, and R. Maboudian, Langmuir 24 (19), 10646 (2008). 
[25] N.-P. Huang, R. Michel, J. Voros, M. Textor, R. Hofer, A. Rossi, D. L. Elbert, J. A. Hubbell, and N. D. Spencer, Langmuir 17 (2), 489 (2001).

[26] N.-P. Huang, J. Vörös, S. M. De Paul, M. Textor, and N. D. Spencer, Langmuir 18 (1), 220 (2002).

[27] M. P. Schwartz, F. Cunin, R. W. Cheung, and M. J. Sailor, Phys. Status Solidi A 202 (8), 1380 (2005).

[28] R. Marie, A. B. Dahlin, J. O. Tegenfeldt, and F. Hook, Biointerphases 2 (1), 49 (2007).

[29] B. Drapp, J. Piehler, A. Brecht, G. Gauglitz, B. J. Luff, J. S. Wilkinson, and J. Ingenhoff, Sens. Actuators B 39 (1-3), 277 (1997).

[30] B. J. Luff, J. S. Wilkinson, J. Piehler, U. Hollenbach, J. Ingenhoff, and N. Fabricius, J. Lightwave. Technol. 16 (4), 583 (1998).

[31] Z.-m. Qi, N. Matsuda, K. Itoh, M. Murabayashi, and C. R. Lavers, Sens. Actuators B 81 (2-3), 254 (2002).

[32] A. Crespi, Y. Gu, B. Ngamsom, H. J. W. M. Hoekstra, C. Dongre, M. Pollnau, R. Ramponi, H. H. van den Vlekkert, P. Watts, G. Cerullo, and R. Osellame, Lab Chip 10 (9), 1167 (2010).

[33] A. Dér, S. Valkai, A. Mathesz, I. Andó, E. K. Wolff, and P. Ormos, Sens. Actuators B 151 (1), 26 (2010).

[34] B. Y. Shew, C. H. Kuo, Y. C. Huang, and Y. H. Tsai, Sens. Actuators A 120 (2), 383 (2005).

[35] B. Y. Shew, Y. C. Cheng, and Y. H. Tsai, Sens. Actuators A 141 (2), 299 (2008).

[36] L. M. Lechuga, A. T. M. Lenferink, R. P. H. Kooyman, and J. Greve, Sens. Actuators B 25 (1-3), 762 (1995).

[37] E. F. Schipper, A. M. Brugman, C. Dominguez, L. M. Lechuga, R. P. H. Kooyman, and J. Greve, Sens. Actuators B 40 (2-3), 147 (1997).

[38] R. G. Heideman and P. V. Lambeck, Sens. Actuators B 61 (1-3), 100 (1999).

[39] F. Brosinger, H. Freimuth, M. Lacher, W. Ehrfeld, E. Gedig, A. Katerkamp, F. Spener, and K. Cammann, Sens. Actuators B 44 (1-3), 350 (1997).

[40] M. Weisser, G. Tovar, S. Mittler-Neher, W. Knoll, F. Brosinger, H. Freimuth, M. Lacher, and W. Ehrfeld, Biosens. Bioelectron. 14 (4), 405 (1999).

[41] F. Prieto, B. Sepulveda, A. Calle, A. Llobera, C. Dominguez, A. Abad, A. Montoya, and L. M. Lechuga, Nanotechnology 14 (8), 907 (2003).

[42] F. Prieto, A. Llobera, D. Jimenez, C. Domenguez, A. Calle, and L. M. Lechuga, J. Lightwave Technol. 18 (7), 966 (2000).

[43] F. Prieto, L. M. Lechuga, A. Calle, A. Llobera, and C. Dominguez, J. Lightwave Technol. 19 (1), 75 (2001). 
[44] K. Zinoviev, Laura G. Carrascosa, Jose Sanchez del Rio, Borja Sepulveda, Carlos Dominguez, and L. M. Lechuga, Adv. Opt. Tech. 2008 ( Article ID: 383927), 6 p (2008).

[45] F. Prieto, B. Sepúlveda, A. Calle, A. Llobera, C. Domínguez, and L. M. Lechuga, Sens. Actuators B 92 (1-2), 151 (2003).

[46] L. M. Lechuga, E. Mauriz, B. Sepúlveda, J. Sánchez del Río, A. Calle, G. Armelles, and C. Dominguez, in Proceedings of the NATO Advanced Research Workshop on Frontiers in Planar Lightwave Circuit Technology, edited by Janz S., J. Ctyroki, and s. Tanev (Kluwer, Otawa, Canada, 2006), Vol. 216, pp. 287.

[47] A. Brandenburg, R. Krauter, C. Künzel, M. Stefan, and H. Schulte, Appl. Opt. 39 (34), 6396 (2000).

[48] K. Schmitt, B. Schirmer, C. Hoffmann, A. Brandenburg, and P. Meyrueis, Biosens. Bioelectron. 22 (11), 2591 (2007).

[49] E. Brynda, M. Houska, A. Brandenburg, and A. Wikerstål, Biosens. Bioelectron. 17 (8), 665 (2002).

[50] C. Hoffmann, K. Schmitt, A. Brandenburg, and S. Hartmann, Anal. Bioanal. Chem. 387 (5), 1921 (2007).

[51] T. Nagel, E. Ehrentreich-Förster, M. Singh, K. Schmitt, A. Brandenburg, A. Berka, and F. F. Bier, Sens. Actuators B 129 (2), 934 (2008).

[52] D. Hradetzky, C. Mueller, and H. Reinecke, J. Opt. A: Pure Appl. Opt. 8 (7), S360 (2006).

[53] A. Ymeti, J. S. Kanger, J. Greve, P. V. Lambeck, R. Wijn, and R. G. Heideman, Appl. Opt. 42 (28), 5649 (2003).

[54] A. Ymeti, J. Greve, P. V. Lambeck, R. Wijn, R. G. Heideman, and J. S. Kanger, Appl. Opt. 44 (17), 3409 (2005).

[55] A. Ymeti, J. S. Kanger, J. Greve, G. A. J. Besselink, P. V. Lambeck, R. Wijn, and R. G. Heideman, Biosens. Bioelectron. 20 (7), 1417 (2005).

[56] A. Ymeti, J. Greve, P. V. Lambeck, T. Wink, S. W. F. M. van Hovell, T. A. M. Beumer, R. R. Wijn, R. G. Heideman, V. Subramaniam, and J. S. Kanger, Nano. Lett. 7 (2), 394 (2007).

[57] B. H. Schneider, J. G. Edwards, and N. F. Hartman, Clin. Chem. 43 (9), 1757 (1997).

[58] B. H. Schneider, E. L. Dickinson, M. D. Vach, J. V. Hoijer, and L. V. Howard, Biosens. Bioelectron. 15 (1-2), 13 (2000).

[59] B. H. Schneider, E. L. Dickinson, M. D. Vach, J. V. Hoijer, and L. V. Howard, Biosens. Bioelectron. 15 (11-12), 597 (2000).

[60] J. Xu, D. Suarez, and D. Gottfried, Anal. Bioanal. Chem. 389 (4), 1193 (2007). 
[61] K. E. Zinoviev, A. B. González-Guerrero, C. Domínguez , and L. M. Lechuga, J. Lightwave Technol. 29 (13), 1926 (2011).

[62] K. Erdélyi, A. G. Frutos, J. J. Ramsden, I. Szendro", and G. Voirin, Grating-Based Optical Biosensors. (John Wiley \& Sons, Ltd, 2008).

[63] J. Vörös, J. J. Ramsden, G. Csúcs, Szendro, I., S. M. De Paul, M. Textor, and N. D. Spencer, Biomaterials 23 (17), 3699 (2002).

[64] P. M. Nellen, K. Tiefenthaler, and W. Lukosz, Sens. Actuators 15 (3), 285 (1988).

[65] P. M. Nellen and W. Lukosz, Sens. Actuators B 1 (1-6), 592 (1990).

[66] W. Lukosz, D. Clerc, P. M. Nellen, C. Stamm, and P. Weiss, Biosens. Bioelectron. 6 (3), 227 (1991).

[67] W. Lukosz, P. M. Nellen, C. Stamm, and P. Weiss, Sens. Actuators B 1 (1-6), 585 (1990).

[68] F. F. Bier, R. Jockers, and R. D. Schmid, Analyst 119 (3), 437 (1994).

[69] F. F. Bier and R. D. Schmid, Biosens. Bioelectron. 9 (2), 125 (1994).

[70] R. Polzius, F. F. Bier, U. Bilitewski, V. Jäger, and R. D. Schmid, Biotechnol. Bioeng. 42 (11), 1287 (1993).

[71] R. Polzius, T. Schneider, F. F. Biert, U. Bilitewski, and W. Koschinski, Biosens. Bioelectron. 11 (5), 503 (1996).

[72] R. Polzius, E. Dießel, F. F. Bier, and U. Bilitewski, Anal. Biochem. 248 (2), 269 (1997).

[73] A. Brandenburg, R. Polzius, F. Bier, U. Bilitewski, and E. Wagner, Sens. Actuators B 30 (1), 55 (1996).

[74] J. Piehler, A. Brandenburg, A. Brecht, E. Wagner, and G. Gauglitz, Appl. Opt. 36 (25), 6554 (1997).

[75] F. F. Bier and F. W. Scheller, Biosens. Bioelectron. 11 (6-7), 669 (1996).

[76] F. F. Bier, F. Kleinjung, and F. W. Scheller, Sens. Actuators B 38 (1-3), 78 (1997).

[77] S. Grego, S. Naskar, A. M. Patel, A. Huffman, C. A. Bower, and B. R. Stoner, Proc. SPIE 6123, 61230D (2006).

[78] S. Grego, J. R. McDaniel, and B. R. Stoner, Sens. Actuators B 131 (2), 347 (2008).

[79] S. Grego, Y. Cao, C. A. Bower, B. R. Stoner, and T. J. Suleski, in Integrated Optics: Devices, Materials, and Technologies XI, edited by Y. Sidorin and C. A. Waechter (Spie-Int Soc Optical Engineering, Bellingham, 2007), Vol. 6475, pp. 47504.

[80] P. K. Yuen, N. H. Fontaine, M. A. Quesada, P. Mazumder, R. Bergman, and E. J. Mozdy, Lab Chip 5 (9), 959 (2005).

[81] M. Wiki and R. E. Kunz, Opt. Lett. 25 (7), 463 (2000).

[82] K. Cottier, M. Wiki, G. Voirin, H. Gao, and R. E. Kunz, Sens. Actuators B 91 (1-3), 241 (2003). 
[83] J. Adrian, S. Pasche, J. M. Diserens, F. Sanchez-Baeza, H. Gao, M. P. Marco, and G. Voirin, Biosens. Bioelectron. 24 (11), 3340 (2009).

[84] J. Adrian, S. Pasche, D. G. Pinacho, H. Font, J. M. Diserens, F. Sanchez-Baeza, B. Granier, G. Voirin, and M. P. Marco, TrAC, Trends Anal. Chem. 28 (6), 769 (2009).

[85] G. Suarez, Y. H. Jin, J. Auerswald, S. Berchtold, H. F. Knapp, J. M. Diserens, Y. Leterrier, J. A. E. Manson, and G. Voirin, Lab Chip 9 (11), 1625 (2009).

[86] S. Pasche, M. Giazzon, B. Wenger, G. Franc, R. Ischer, G. J. Oostingh, and G. Voirin, Proced. Chem. 1 (1), 738 (2009).

[87] J. Razumovitch, K. de Franca, F. Kehl, M. Wiki, W. Meier, and C. Vebert, J. Phys. Chem. B 113 (24), 8383 (2009).

[88] R. W. Boyd and J. E. Heebner, Appl. Opt. 40 (31), 5742 (2001).

[89] S. Lee, S. C. Eom, J. S. Chang, C. Huh, G. Y. Sung, and J. H. Shin, Opt. Express 18 (20), 20638 (2010).

[90] S. Lee, S. C. Eom, J. S. Chang, C. Huh, G. Y. Sung, and J. H. Shin, Opt. Express 18 (11), 11209 (2010).

[91] A. Schweinsberg, S. Hocdé, N. N. Lepeshkin, R. W. Boyd, C. Chase, and J. E. Fajardo, Sens. Actuators B 123 (2), 727 (2007).

[92] K. De Vos, I. Bartolozzi, E. Schacht, P. Bienstman, and R. Baets, Opt. Express 15 (12), 7610 (2007).

[93] K. M. De Vos, I. Bartolozzi, P. Bienstman, R. Baets, and E. Schacht, XXX P Soc Photo-Opt Ins 6447, K4470 (2007).

[94] X. Li, Z. Zhang, S. Qin, T. Wang, F. Liu, M. Qiu, and Y. Su, Appl. Opt. 48 (25), F90 (2009).

[95] T. Claes, J. G. Molera, K. De Vos, E. Schacht, R. Baets, and P. Bienstman, IEEE Photonics J. 1 (3), 197 (2009).

[96] M. Iqbal, M. A. Gleeson, B. Spaugh, F. Tybor, W. G. Gunn, M. Hochberg, T. BaehrJones, R. C. Bailey, and L. C. Gunn, IEEE J. Sel. Top. Quant. 16 (3), 654 (2010).

[97] M. S. Luchansky, A. L. Washburn, T. A. Martin, M. Iqbal, L. C. Gunn, and R. C. Bailey, Biosens. Bioelectron. 26 (4), 1283 (2010).

[98] A. M. Armani, R. P. Kulkarni, S. E. Fraser, R. C. Flagan, and K. J. Vahala, Science 317 (5839), 783 (2007).

[99] A. M. Armani, A. Srinivasan, and K. J. Vahala, Nano. Lett. 7 (6), 1823 (2007).

[100] A. M. Armani and K. J. Vahala, Biophys. J., 29a (2007).

[101] H. K. Hunt, C. Soteropulos, and A. M. Armani, Sensors 10 (10), 9317 (2010).

[102] X. M. Zhang, H. S. Choi, and A. M. Armani, Appl. Phys. Lett. 96 (15) (2010). 
[103] A. Yalcin, K. C. Popat, J. C. Aldridge, T. A. Desai, J. Hryniewicz, N. Chbouki, B. E. Little, K. Oliver, V. Van, C. Sai, D. Gill, M. Anthes-Washburn, M. S. Unlu, and B. B. Goldberg, IEEE J. Sel. Top. Quantum Electron. 12 (1), 148 (2006).

[104] A. Ramachandran, S. Wang, J. Clarke, S. J. Ja, D. Goad, L. Wald, E. M. Flood, E. Knobbe, J. V. Hryniewicz, S. T. Chu, D. Gill, W. Chen, O. King, and B. E. Little, Biosens. Bioelectron. 23 (7), 939 (2008).

[105] A. Ksendzov and Y. Lin, Opt. Lett. 30 (24), 3344 (2005).

[106] P. Rabiei and W. H. Steier, presented at the Lasers and Electro-Optics Society, 2001. LEOS 2001. The 14th Annual Meeting of the IEEE, 2001 (unpublished).

[107] M. Jazbinsek, P. Rabiei, C. Bosshard, and P. Gunter, XXX Aip Conf Proc 709, 187 (2004).

[108] A. Chen, L. Dalton, T. Sherwood, A. K. Y. Jen, P. Rabiei, W. Steier, Y. Huang, G. T. Paloczi, J. K. S. Poon, A. Scherer, and A. Yariv, XXX P Soc Photo-Opt Ins 5708, 187 (2005).

[109] C.-Y. Chao and L. J. Guo, Appl. Phys. Lett. 83 (8), 1527 (2003).

[110] R. Dekker and et al., J. Phys. D. Appl. Phys. 40 (14), R249 (2007).

[111] K. De Vos, J. Girones, S. Popelka, E. Schacht, R. Baets, and P. Bienstman, Biosens. Bioelectron. 24 (8), 2528 (2009).

[112] K. De Vos, J. Girones, T. Claes, Y. De Koninck, S. Popelka, E. Schacht, R. Baets, and P. Bienstman, IEEE Photonics J. 1 (4), 225 (2009).

[113] J. Y. Byeon, F. T. Limpoco, and R. C. Bailey, Langmuir 26 (19), 15430 (2010).

[114] M. S. Luchansky and R. C. Bailey, Anal. Chem. 82 (5), 1975 (2010).

[115] A. L. Washburn, L. C. Gunn, and R. C. Bailey, Anal. Chem. 81 (22), 9499 (2009).

[116] A. L. Washburn, M. S. Luchansky, A. L. Bowman, and R. C. Bailey, Anal. Chem. 82 (1), 69 (2010).

[117] A. J. Qavi and R. C. Bailey, Angew. Chem. Int. Ed. 49 (27), 4608 (2010).

[118] D. K. Armani, T. J. Kippenberg, S. M. Spillane, and K. J. Vahala, Nature 421 (6926), 925 (2003).

[119] A. M. Armani, D. K. Armani, B. Min, K. J. Vahala, and S. M. Spillane, Appl. Phys. Lett. 87 (15) (2005).

[120] H. S. Choi, X. M. Zhang, and A. M. Armani, Opt. Lett. 35 (4), 459 (2010).

[121] R. V. Nair and R. Vijaya, Prog. Quantum Electron. 34 (3), 89 (2010).

[122] J. D. Joannopoulos, S. G. Johnson, J. N. Winn, and R. D. Meade, Photonic Crystals. Moding the Flow of Light. (Princeton University Press, 2008).

[123] I. D. Block, N. Ganesh, M. Lu, and B. T. Cunningham, IEEE Sens. J. 8 (3-4), 274 (2008).

[124] B. Cunningham, P. Li, B. Lin, and J. Pepper, Sens. Actuators B 81 (2-3), 316 (2002). 
[125] B. Cunningham, B. Lin, J. Qiu, P. Li, J. Pepper, and B. Hugh, Sens. Actuators B 85 (3), 219 (2002).

[126] B. Cunningham, J. Qiu, P. Li, and B. Lin, Sens. Actuators B 87 (2), 365 (2002).

[127] I. D. Block, L. L. Chan, and B. T. Cunningham, Sens. Actuators B 120 (1), 187 (2006).

[128] B. T. Cunningham, P. Li, S. Schulz, B. Lin, C. Baird, J. Gerstenmaier, C. Genick, F. Wang, E. Fine, and L. Laing, J. Biomol. Screening 9 (6), 481 (2004).

[129] C. J. Choi and B. T. Cunningham, Lab Chip 6 (10), 1373 (2006).

[130] C. J. Choi and B. T. Cunningham, Lab Chip 7 (5), 550 (2007).

[131] C. J. Choi, I. D. Block, B. Bole, D. Dralle, and B. T. Cunningham, IEEE Sens. J. 9 (12), 1697 (2009).

[132] C. J. Choi, A. R. Belobraydich, L. L. Chan, P. C. Mathias, and B. T. Cunningham, Anal. Biochem. 405 (1), 1 (2010).

[133] B. R. Schudel, C. J. Choi, B. T. Cunningham, and P. J. A. Kenis, Lab Chip 9 (12), 1676 (2009).

[134] B. T. Cunningham and L. Laing, Expert Rev. Proteomics 3 (3), 271 (2006).

[135] B. Lin, P. Li, and B. T. Cunningham, Sens. Actuators B 114 (2), 559 (2006).

[136] L. L. Chan, S. L. Gosangari, K. L. Watkin, and B. T. Cunningham, Apoptosis 12 (6), 1061 (2007).

[137] L. L. Chan, S. L. Gosangari, K. L. Watkin, and B. T. Cunningham, Sens. Actuators B 132 (2), 418 (2008).

[138] M. F. Pineda, L. L. Y. Chan, T. Kuhlenschmidt, C. J. Choi, M. Kuhlenschmidt, and B. T. Cunningham, IEEE Sens. J. 9 (4), 470 (2009).

[139] L. L. Chan, E. A. Lidstone, K. E. Finch, J. T. Heeres, P. J. Hergenrother, and B. T. Cunningham, Jala 14 (6), 348 (2009).

[140] J. T. Heeres, S. H. Kim, B. J. Leslie, E. A. Lidstone, B. T. Cunningham, and P. J. Hergenrother, J. Am. Chem. Soc. 131 (51), 18202 (2009).

[141] T. Xu, N. Zhu, M. Y. C. Xu, L. Wosinski, J. S. Aitchison, and H. E. Ruda, Opt. Express 18 (6), 5420 (2010).

[142] D. F. Dorfner, T. Hurlimann, T. Zabel, L. H. Frandsen, G. Abstreiter, and J. J. Finley, Appl. Phys. Lett. 93 (18), 181103 (2008).

[143] D. Dorfner, T. Zabel, T. Hurlimann, N. Hauke, L. Frandsen, U. Rant, G. Abstreiter, and J. Finley, Biosens. Bioelectron. 24 (12), 3688 (2009).

[144] N. Skivesen, A. Tetu, M. Kristensen, J. Kjems, L. H. Frandsen, and P. I. Borel, Opt. Express 15 (6), 3169 (2007).

[145] J. Garcia-Ruperez, V. Toccafondo, M. J. Banuls, J. G. Castello, A. Griol, S. PeransiLlopis, and A. Maquieira, Opt. Express 18 (23), 24276 (2010). 
[146] V. Toccafondo, J. García-Rupérez, M. J. Bañuls, A. Griol, J. G. Castelló, S. PeransiLlopis, and A. Maquieira, Opt. Lett. 35 (21), 3673 (2010).

[147] J. S. Foresi, P. R. Villeneuve, J. Ferrera, E. R. Thoen, G. Steinmeyer, S. Fan, J. D. Joannopoulos, L. C. Kimerling, H. I. Smith, and E. P. Ippen, Nature 390 (6656), 143 (1997).

[148] S. Mandal and D. Erickson, Opt. Express 16 (3), 1623 (2008).

[149] S. Mandal, R. Akhmechet, L. Chen, S. Nugen, A. Baeumner, and D. Erickson, Proced. SPIE 6645, 6451J (2007).

[150] S. Mandal, J. M. Goddard, and D. Erickson, Lab Chip 9 (20), 2924 (2009).

[151] M. Lee and P. M. Fauchet, Opt. Express 15 (8), 4530 (2007).

[152] E. Guillermain and P. M. Fauchet, Proc. SPIE 7167, 71670D (2009).

[153] S. Pal, E. Guillermain, R. Sriram, B. L. Miller, and P. M. Fauchet, Biosens. Bioelectron. 26 (10), 4024 (2011).

[154] S. Zlatanovic, L. W. Mirkarimi, M. M. Sigalas, M. A. Bynum, E. Chow, K. M. Robotti, G. W. Burr, S. Esener, and A. Grot, Sens. Actuators B 141 (1), 13 (2009).

[155] M. R. Lee and P. M. Fauchet, Opt. Lett. 32 (22), 3284 (2007).

[156] S. Mandal, X. Serey, and D. Erickson, Nano. Lett. 10 (1), 99 (2010).

[157] D. Erickson, X. Serey, Y.-F. Chen, and S. Mandal, Lab Chip 11 (6), 995 (2011).

[158] A. Densmore, D. X. Xu, P. Waldron, S. Janz, P. Cheben, J. Lapointe, A. Delage, B. Lamontagne, J. H. Schmid, and E. Post, IEEE Phot. Tech. Lett. 18 (21-24), 2520 (2006).

[159] A. Densmore, D. X. Xu, S. Janz, P. Waldron, T. Mischki, G. Lopinski, A. Delage, J. Lapointe, P. Cheben, B. Lamontagne, and J. H. Schmid, Opt. Lett. 33 (6), 596 (2008).

[160] D. X. Xu, M. Vachon, A. Densmore, R. Ma, S. Janz, A. Delage, J. Lapointe, P. Cheben, J. H. Schmid, E. Post, S. Messaoudene, and J. M. Fedeli, Opt. Express 18 (22), 22867 (2010).

[161] A. Densmore, M. Vachon, D. X. Xu, S. Janz, R. Ma, Y. H. Li, G. Lopinski, A. Delage, J. Lapointe, C. C. Luebbert, Q. Y. Liu, P. Cheben, and J. H. Schmid, Opt. Lett. 34 (23), 3598 (2009).

[162] D. X. Xu, M. Vachon, A. Densmore, R. Ma, A. Delage, S. Janz, J. Lapointe, Y. Li, G. Lopinski, D. Zhang, Q. Y. Liu, P. Cheben, and J. H. Schmid, Opt. Lett. 35 (16), 2771 (2010).

[163] V. R. Almeida, Q. Xu, C. A. Barrios, and M. Lipson, Opt. Lett. 29 (11), 1209 (2004).

[164] F. Dell'Olio and V. M. N. Passaro, Opt. Express 15 (8), 4977 (2007).

[165] C. A. Barrios, Sensors 9 (6), 4751 (2009).

[166] C. A. Barrios, K. B. Gylfason, B. Sanchez, A. Griol, H. Sohlstrom, M. Holgado, and R. Casquel, Opt. Lett. 32 (21), 3080 (2007). 
[167] C. A. Barrios, M. J. Banuls, V. Gonzalez-Pedro, K. B. Gylfason, B. Sanchez, A. Griol, A. Maquieira, H. Sohlstrom, M. Holgado, and R. Casquel, Opt. Lett. 33 (7), 708 (2008).

[168] C. F. Carlborg, K. B. Gylfason, A. Kazmierczak, F. Dortu, M. J. B. Polo, A. Maquiera Catala, G. M. Kresbach, H. Sohlstrom, T. Moh, L. Vivien, J. Popplewell, G. Ronan, C. A. Barrios, G. Stemme, and W. van der Wijngaart, Lab Chip 10 (3), 281 (2010).

[169] K. B. Gylfason, C. F. Carlborg, A. Kazmierczak, F. Dortu, H. Sohlstrom, L. Vivien, C. A. Barrios, W. van der Wijngaart, and G. Stemme, Opt. Express 18 (4), 3226 (2010).

[170] A. D. Falco, L. O'Faolain, and T. F. Krauss, Appl. Phys. Lett. 92 (8), 083501 (2008).

[171] A. Di Falco, L. O'Faolain, and T. F. Krauss, Appl. Phys. Lett. 94 (6), 063503 (2009).

[172] M. Holgado, R. Casquel, B. Sanchez, C. Molpeceres, M. Morales, and J. L. Ocana, Opt. Express 15 (20), 13318 (2007).

[173] D. Lopez-Romero, C. A. Barrios, M. Holgado, M. F. Laguna, and R. Casquel, Microelectron. Eng. 87 (4), 663 (2010).

[174] M. Holgado, R. Casquel, C. Molpeceres, M. Morales, and J. L. Ocana, Sensor Lett. 6 (4), 564 (2008).

[175] M. Holgado, C. A. Barrios, F. J. Ortega, F. J. Sanza, R. Casquel, M. F. Laguna, M. J. Banuls, D. Lopez-Romero, R. Puchades, and A. Maquieira, Biosens. Bioelectron. 25 (12), 2553 (2010).

[176] T. Braun, M. K. Ghatkesar, N. Backmann, W. Grange, P. Boulanger, L. Letellier, H.-P. Lang, A. Bietsch, C. Gerber, and M. Hegner, Nat. Nanotechnol. 4 (3), 179 (2009).

[177] A. Salehi-Khojin, S. Bashash, N. Jalili, M. Muller, and R. Berger, J. Appl. Phys. 105 (1), 013506 (2009).

[178] J. W. Ndieyira, M. Watari, A. D. Barrera, D. Zhou, M. Vogtli, M. Batchelor, M. A. Cooper, T. Strunz, M. A. Horton, C. Abell, T. Rayment, G. Aeppli, and R. A. McKendry, Nat. Nanotechnol. 3 (11), 691 (2008).

[179] M. Alvarez, A. Calle, J. Tamayo, L. M. Lechuga, A. Abad, and A. Montoya, Biosens. Bioelectron. 18 (5-6), 649 (2003).

[180] M. Alvarez and L. M. Lechuga, Analyst 135 (5), 827 (2010).

[181] A. K. Naik, M. S. Hanay, W. K. Hiebert, X. L. Feng, and M. L. Roukes, Nat. Nanotechnol. 4, 445 (2009).

[182] N. Privorotskaya, Y.-S. Liu, J. Lee, H. Zeng, J. A. Carlisle, A. Radadia, L. Millet, R. Bashir, and W. P. King, Lab Chip 10 (9), 1135 (2010).

[183] S. Singamaneni, M. C. LeMieux, H. P. Lang, C. Gerber, Y. Lam, S. Zauscher, P. G. Datskos, N. V. Lavrik, H. Jiang, R. R. Naik, T. J. Bunning, and V. V. Tsukruk, Adv. Mater. 20 (4), 653 (2008).

[184] S. W. Stahl, E. M. Puchner, and H. E. Gaub, Rev. Sci. Instrum. 80 (7), 073702 (2009).

[185] D. Rugar, R. Budakian, H. J. Mamin, and B. W. Chui, Nature 430 (6997), 329 (2004). 
[186] K. Zinoviev, C. Dominguez, J. A. Plaza, V. c. J. C. Busto, and L. M. Lechuga, J. Lightwave Technol. 24 (5), 2132 (2006).

[187] J. W. Noh, R. R. Anderson, S. Kim, W. Hu, and G. P. Nordin, Opt. Express 17 (22), 20012 (2009).

[188] J. W. Noh, R. R. Anderson, S. Kim, W. Hu, and G. P. Nordin, Nanotechnology 21 (15), 155501 (2010).

[189] R. Panergo, C.-S. Huang, C.-S. Liu, P. G. Reinhall, and W.-C. Wang, J. Lightwave Technol. 25 (3), 850 (2007).

[190] T. Xu, M. Bachman, F.-G. Zeng, and G.-P. Li, Sens. Actuators A 114 (2-3), 176 (2004).

[191] S. T. Koev, R. Fernandes, W. E. Bentley, and R. Ghodssi, IEEE T. Biomed. Circ. S. 3 (6), 415 (2009).

[192] M. Nordstrom, D. A. Zauner, A. Boisen, and J. Hubner, J. Lightwave Technol. 25 (5), 1284 (2007).

[193] A. Llobera, V. J. Cadarso, K. Zinoviev, C. Dominguez, S. Buttgenbach, J. Vila, J. A. Plaza, and S. Biittgenbach, IEEE Photon. Technol. Lett. 21 (2), 79 (2009).

[194] M. W. Pruessner, N. Siwak, K. Amarnath, S. Kanakaraju, W.-H. Chuang, and R. Ghodssi, J. Micromech. Microeng. 16 (4), 832 (2006).

[195] C. Lee, J. Thillaigovindan, C.-C. Chen, X. T. Chen, Y.-T. Chao, S. Tao, W. Xiang, A. Yu, H. Feng, and G. Q. Lo, in Appl. Phys. Lett. (2008).

[196] W. H. P. Pernice, M. Li, and H. X. Tang, Opt. Express 17 (15), 12424 (2009).

[197] W. Xiang and C. Lee, IEEE J. Sel. Top. Quantum Electron. 15 (5), 1323 (2009).

[198] M. W. Pruessner, T. H. Stievater, M. S. Ferraro, W. S. Rabinovich, J. L. Stepnowski, and R. A. McGill, Lab Chip 10 (6), 762 (2010).

[199] K. Zinoviev, C. Dominguez, and A. Vila, Opt. Express 13 (21), 8618 (2005).

[200] W. S. Hu, R. Anderson, Y. S. Qian, J. G. Song, J. W. Noh, S. Kim, and G. P. Nordin, Rev. Sci. Instrum. 80 (8) (2009).

[201] K. Misiakos, I. Raptis, A. Gerardino, H. Contopanagos, and M. Kitsara, Lab Chip 9 (9), 1261 (2009).

[202] M. Li, P. H. P., and T. X., Nat. Photonics 3 (8), 464 (2009).

[203] M. Li, W. H. P. Pernice, C. Xiong, T. Baehr-Jones, M. Hochberg, and H. X. Tang, Nature 456 (7221), 480 (2008).

[204] R. R. Anderson, W. Hu, J. W. Noh, W. C. Dahlquist, S. J. Ness, T. M. Gustafson, D. C. Richards, S. Kim, B. A. Mazzeo, A. T. Woolley, and G. P. Nordin, Lab Chip 11 (12), 2088 (2011).

[205] M. D. LaHaye, O. Buu, B. Camarota, and K. C. Schwab, Science 304 (5667), 74 (2004).

[206] K. C. Schwab and M. L. Roukes, Phys. Today 58 (7), 36 (2005). 
[207] J. D. Teufel, T. Donner, M. A. Castellanos-Beltran, J. W. Harlow, and K. W. Lehnert, Nat. Nanotechnol. 4 (12), 820 (2009).

[208] J. D. Teufel, J. W. Harlow, C. A. Regal, and K. W. Lehnert, Phys. Rev. Lett. 101 (19), 197203 (2008).

[209] F. J. Blanco, M. Agirregabiria, J. Berganzo, K. Mayora, J. Elizalde, A. Calle, C. Dominguez, and L. M. Lechuga, J. Micromech. Microeng. 16 (5), 1006 (2006).

[210] N. M. Jokerst, L. Luan, S. Palit, M. Royal, S. Dhar, M. A. Brooke, and T. Tyler, IEEE T. Biomed. Circ. S. 3 (4), 202 (2009).

[211] K. B. Mogensen and J. P. Kutter, Electrophoresis 30 (S1), S92 (2009).

[212] J. J. Lillie, M. A. Thomas, N. M. Jokerst, S. E. Ralph, K. A. Dennis, and C. L. Henderson, J. Opt. Soc. Am. B 23 (4), 642 (2006).

[213] in PHYTIA European Union project (www.pythia-project.eu).

[214] R. Osellame, H. J. W. M. Hoekstra, G. Cerullo, and M. Pollnau, Laser Photonic Rev. 5 (3), 442 (2011). 


\section{Abstract Figure}

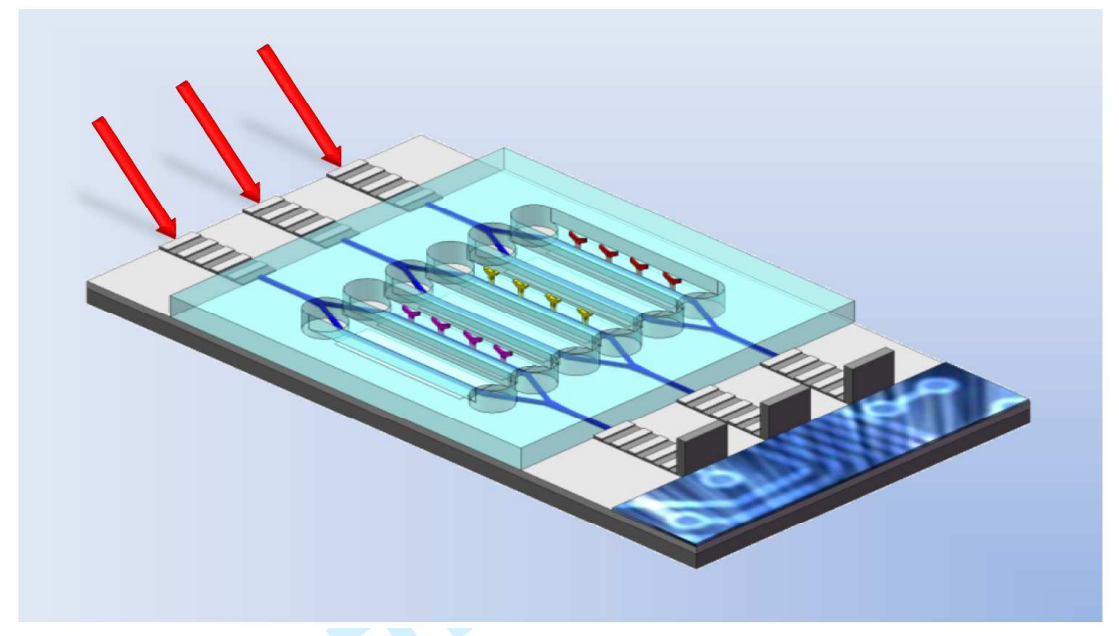

Wiley - VCH 
$\mathrm{M}^{\mathrm{a}}$ Carmen Estevez graduated with a Bachelor of Chemistry in 1998. Subsequently she joined the Spanish National Research Council (CSIC) where she started her research in the field of immunochemistry and bioanalytical chemistry. She obtained her MSc and $\mathrm{PhD}$ in Chemistry from the University of Barcelona in 1999 and 2005, respectively. After her PhD, she joined the University of Florida in 2006 as Postdoctoral Researcher until 2008 where she focused on the production and applications of fluorescent nanoparticles in bioanalytical applications. In 2009 she joined the Nanobiosensors and Bioanalytical Applications group at the Research Center on Nanoscience and Nanotechnology (CIN2) where she is focusing on surface biofunctionalization and clinical diagnostics applications of label-free optical biosensors.

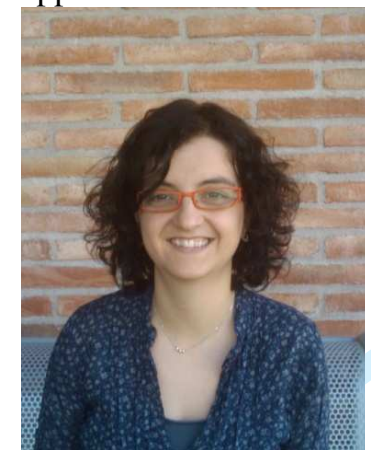

Mar Alvarez is a researcher at the Nanobiosensors and Bioanalytical Applications Group in the Research Center on Nanoscience and Nanotechnology (CIN2) of the Spanish National Research Council (CSIC), with a special interest in biotechnology and nanofabrication for biosensor devices. She is especially involved in the development of nanomechanical-based biosensors and their integration in lab-on-chip platforms for clinical diagnosis. She obtained her MSc and $\mathrm{PhD}$ degrees in Physics from the Autonoma University of Madrid, Spain. After her PhD she joined Monash University (Melbourne, Australia) for a post-doctoral experience.
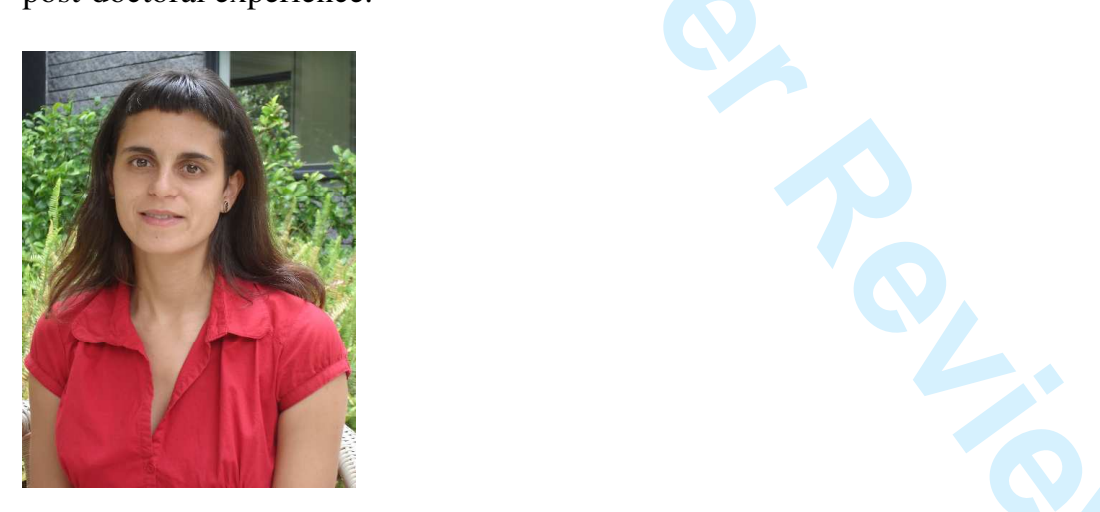

Laura M. Lechuga is Full Professor of the Spanish National Research Council (CSIC). She is the Head of the Nanobiosensors and Bioanalytical Applications Group at the Research Center on Nanoscience and Nanotechnology (CIN2), CSIC. The principal focus of her research program is the development of biosensor devices based on plasmonics, magnetoplasmonics, integrated photonics and nanomechanics principles, including surface biofunctionalization, microfluidics and lab-on-a-chip integration. The biosensing platforms are applied to the environmental control of pollutants, early diagnosis of cancer and diseases and genomics and proteomics.

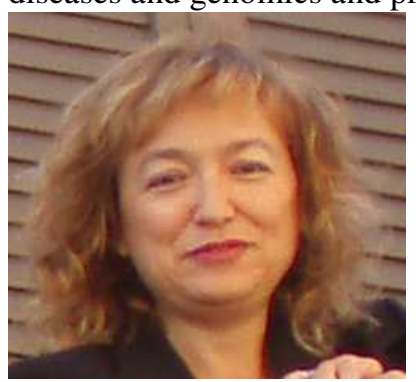


Figure 1. Scheme of the evanescent field sensing. A biomolecular interaction taking place at the waveguide surface within the evanescent region induces a change in the effective refractive index of the transmitted light mode. The evaluation of this optical change gives a measure of the number of molecules which has been detected. $59 \times 32 \mathrm{~mm}(600 \times 600 \mathrm{DPI})$ 
Figure 2. General scheme for the activation of the sensor surface and common strategies for biofunctionalization: surface cleaning (1), chemical activation of plain surface (2), immobilization of the specific bioreceptors (3) and final detection of the target molecule (4). The bioassays depicted in (3) and (4) are the following: (a) Mixed self-assembled monolayer (SAM) with reactive and nonreactive silanes compounds with specific antibodies covalently immobilized for protein recognition;

(b) hydrophilic and biocompatible reactive monolayer based on pegylated-silane or dextran compounds with proteins covalently immobilized for antibody recognition; (c) Affinity tags immobilized on the surface (i.e. protein A, or streptavidin onto biotinylated surface) to achieve appropriate orientation of specific antibodies for protein recognition; (d) covalent immobilization of DNA or RNA probes (together with non-specific spacers) for hybridization with complementary DNA/RNA strands. $99 \times 47 \mathrm{~mm}(300 \times 300 \mathrm{DPI})$ 


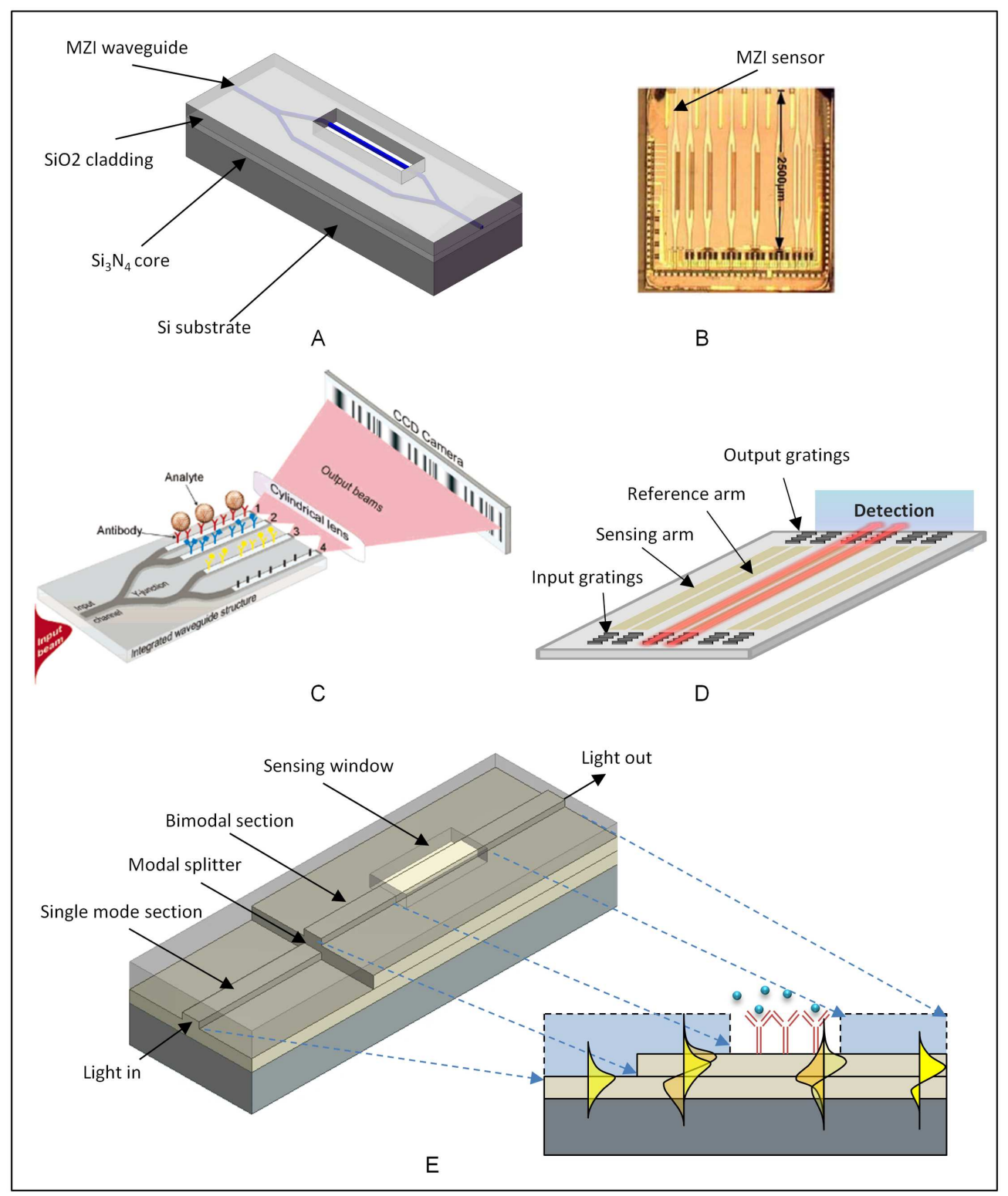

Figure 3. Several interferometric transducers for sensing: (A) Mach-Zehnder interferometer; (B) Mach-Zehnder interferometer sensor array with reference channels, reprinted from [210] with permission from IEEE (C 2009, IEEE); (C) Four Channel Young Interferometer, reprinted with permission from [56]. Copyright 2007 American Chemical Society; (D) Hartman Interferometer; (E) Bimodal Waveguide Interferometer. $189 \times 225 \mathrm{~mm}(300 \times 300 \mathrm{DPI})$ 
A
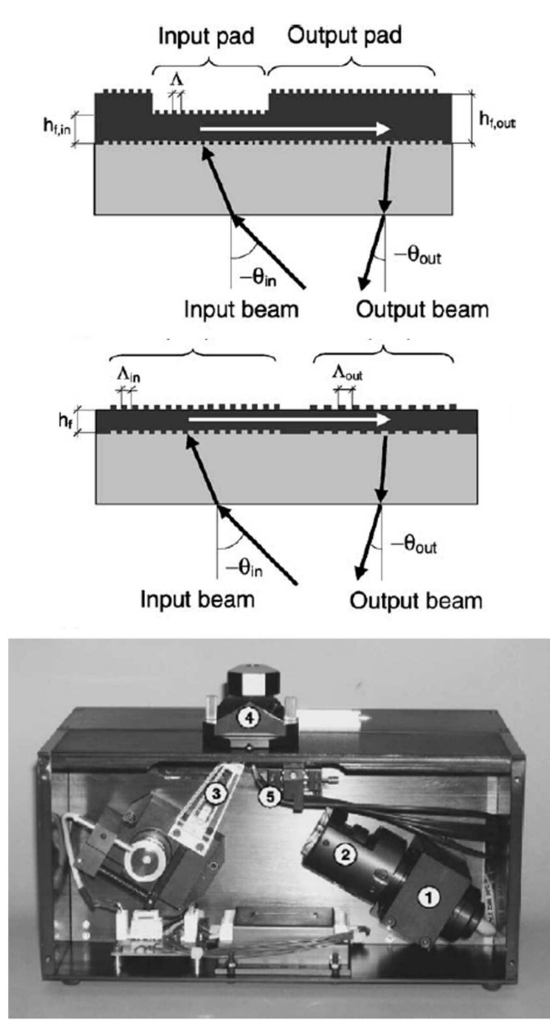

B
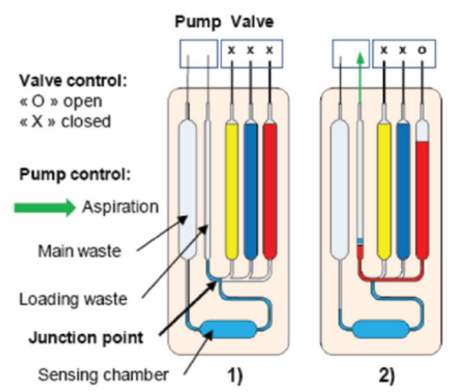

2)
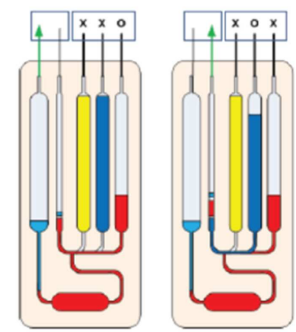

4)

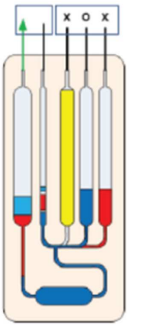

5)

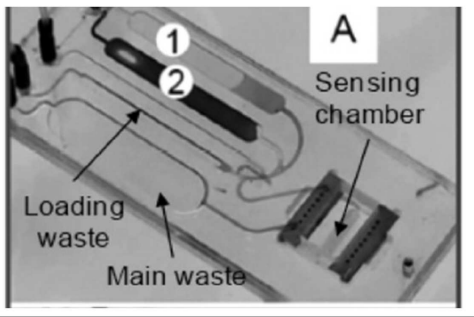

Figure 4. (A) Wavelength Interrogated Optical Sensor (WIOS) based on in and output grating couplers. Two different grating configurations are shown: a grating with different periods for in and output and a single grating with different thickness layer. Photograph of a compact WIOS instrument with (1) laser source, (2) beam expander, (3) deflection mirror, (4) sensing chip with fluidic cell, and (5) array of plastic optical fibers. Reprinted from [82] with permission from Elsevier.

(B) Lab-on-a-chip based on WIOS configuration adapted to a polymer-based self-contained microfluidic cartridge. Adapted and reproduced from [85] with permission of the Royal Society of Chemistry. $129 \times 116 \mathrm{~mm}(600 \times 600 \mathrm{DPI})$ 

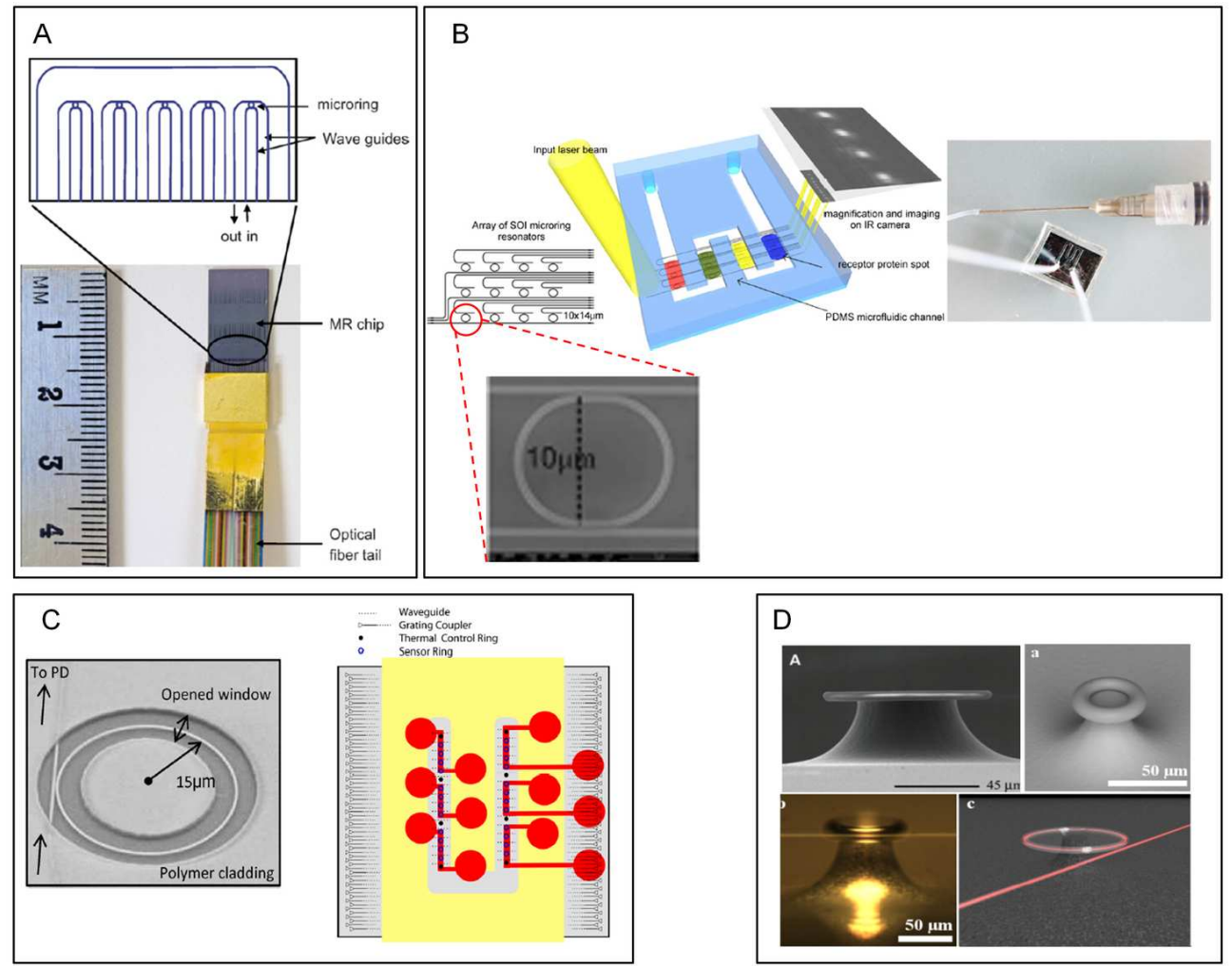

Figure 5. Several resonator sensing configurations: (A) Silicon chip with five rings vertically coupled to input and output port waveguides. Reprinted from [104] with permission from Elsevier. (B) Array platform of SOI-based ring resonators packaged with PDMS microfluidics. Reprinted from [112] with permission from IEEE (C) 2009 IEEE) (C) SEM micrograph of a single ring with a window opened in the cladding polymer to expose the sensor surface. Microchip with 32 sensors and a six-channel PDMS microfluidic cell for multiplexed analyses. Reprinted from [96] and [116] with permission from IEEE (C 2010 IEEE) and from American Chemical Society, respectively. (D) Top: Scanning electron micrographs of microtoroids; Bottom: Microtoroids images showing the tapered fiber for incoupling of the light. Reprinted from [98] with permission from AAAS and from [101]. $124 \times 97 \mathrm{~mm}(300 \times 300 \mathrm{DPI})$ 

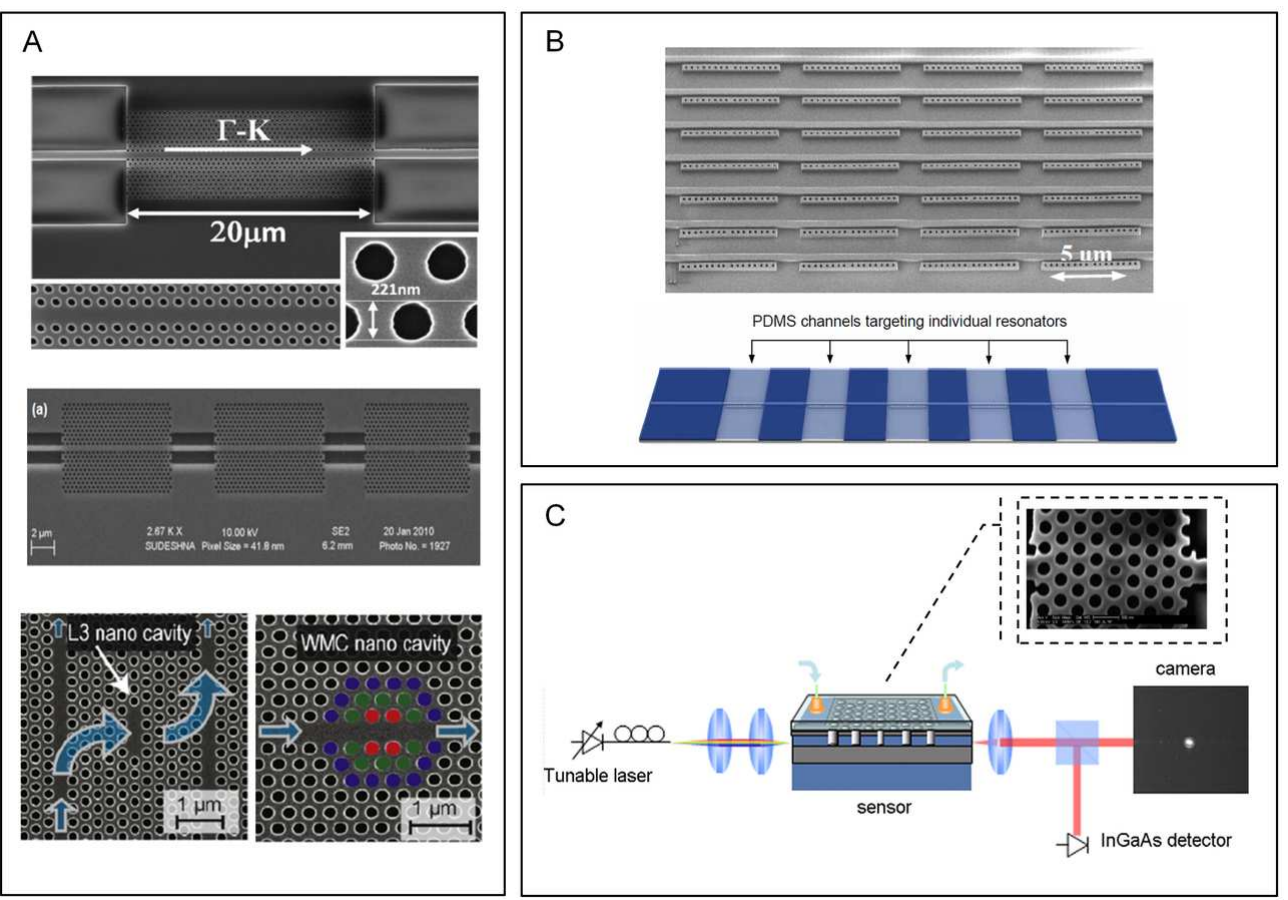

Figure 6. (A) SEM images of several photonic crystal-based waveguides sensors. Reprinted from [146] with permission from the Optical Society of America and from $[143,153]$ with permission from Elsevier. (B) SEM image of a nanoscale optofluidic array of photonic crystal microcavities with multiplexed capabilities (top) and a scheme of a PDMS fluidic channel used for sample delivery (bottom). Reprinted from [148] with permission from the Optical Society of America. (C) Scheme of the evaluation set-up of a photonic crystal microcavity sensor, showing the optics (laser, fiber polarizer, input collimating and focusing optics, output objective, photodetector and alignment camera) and integrated microfluidics. SEM image of the photonic crystal structure is also included. Reprinted from [154] with permission from Elsevier. $109 \times 78 \mathrm{~mm}(300 \times 300$ DPI $)$ 

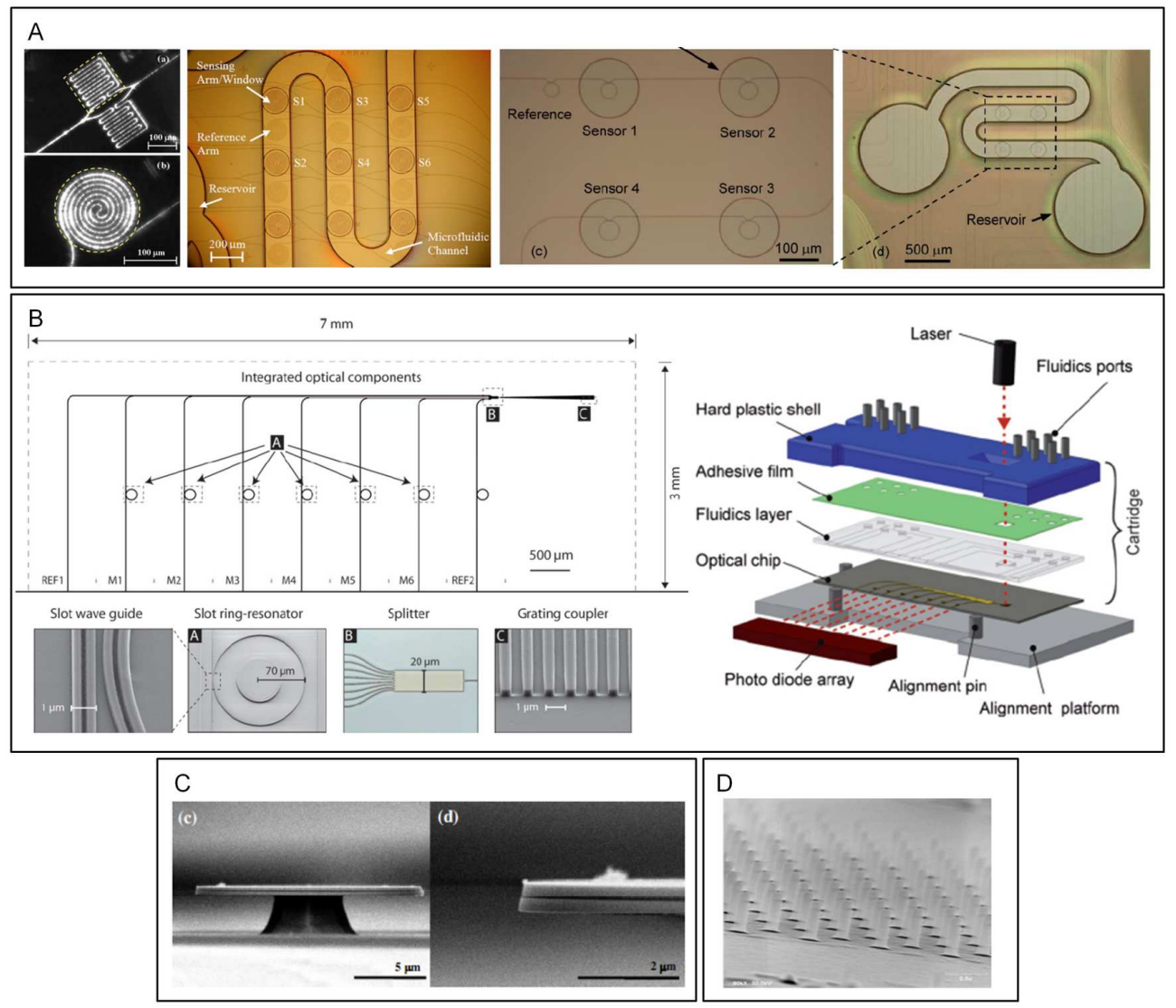

Figure 7. (A) Silicon photonic wire MZI and resonator sensor arrays. Details of the structures and the SU-8 microfluidic channels aligned to the sensor windows are shown. Reprinted from [159-161] with permission from the Optical Society of America. (B) Slot-waveguide ring resonator chip, consisting of 6 sensing channels plus 2 reference ones. Details of the structures and grating couplers of the resonator array (left) and the compact sensor cartridge for multiplexed sensing (right) are shown. Reprinted from [168] with permission of the Royal Society of Chemistry. (C) SEM images of fabricated air-slot disk with a diameter of $15 \mu \mathrm{m}$ and an air slot depth of $2 \mu \mathrm{m}$. Reprinted from [89] with permission from the Optical Society of America. (D) SEM images of SU-8 nanocolumns lattices. Reprinted from [175] with permission from Elsevier. $149 \times 128 \mathrm{~mm}(300 \times 300 \mathrm{DPI})$ 
Figure 8. (A) Schematic representation of the optical waveguide cantilever (OWC) working principle. Input light is coupled to the cantilever through the evanescent field of an input waveguide, and output light is collected in a misaligned waveguide after crossing a small gap. (B) Picture and SEM image of an OWC chip array with 20 waveguide microcantilevers. Reproduced from [180] with permission of the Royal Society of Chemistry. $119 \times 119 \mathrm{~mm}(600 \times 600 \mathrm{DPI})$ 
Figure 9. Envisioned lab-on-a-chip platform with on-chip detection using integrated optics-based transducers. In the figure, an array of Mach-Zehnder interferometers are integrated with individual microfluidics channels, grating couplers for the in and output of the light in each sensing channel, read-out photodetectors and CMOS electronics for data processing. $63 \times 35 \mathrm{~mm}(600 \times 600 \mathrm{DPI})$ 
2

Table 1. Comparison of limit of detection (LOD) in integrated optical biosensors

\begin{tabular}{|c|c|c|c|}
\hline Device & Mass detection limit $\left(\mathrm{pg} / \mathrm{mm}^{2}\right)$ & RI detection limit (RIU) & References \\
\hline $\mathrm{SPR}^{\mathrm{a}}$ & $1-5^{(\mathrm{a}, \mathrm{b})}$ & $10^{-5}-10^{-7}$ & [4] \\
\hline \multicolumn{4}{|l|}{ Grating Couplers } \\
\hline \multirow[t]{2}{*}{ Input and/or/output } & n.d $\mathrm{d}^{(\mathrm{c})}$ & $\sim 2-5 \cdot 10^{-6}$ & {$[64,66]$} \\
\hline & 0.3 & $<10^{-6}$ & [82] \\
\hline Reflected mode & 10 & $3 \cdot 10^{-6}$ & {$[73 ; 74]$} \\
\hline \multicolumn{4}{|l|}{ Interferometer } \\
\hline \multirow[t]{4}{*}{ Mach Zehnder } & n.d & $7 \cdot 10^{-6}$ & [41] \\
\hline & 0.06 & $1 \cdot 10^{-7}$ & [44] \\
\hline & $0.01^{(\mathrm{d})}$ & $2 \cdot 10^{-8(\mathrm{~d})}$ & [12] \\
\hline & n.d. & $9.2 \cdot 10^{-7}$ & [212] \\
\hline \multirow[t]{4}{*}{ Young } & n.d. & $5 \cdot 10^{-6}$ & {$[52]$} \\
\hline & 0.75 & $9 \cdot 10^{-8}$ & [47] \\
\hline & $0.013^{(\mathrm{d})}$ & $9 \cdot 10^{-9}$ & [48] \\
\hline & $0.020^{(\mathrm{d})}$ & $8.5 \cdot 10^{-8}$ & [54] \\
\hline Hartman & n.d & $\sim 10^{-6}$ & [60] \\
\hline Bimodal waveguide & n.d. & $2.5 \cdot 10^{-7}$ & [61] \\
\hline \multicolumn{4}{|l|}{ Microring resonator } \\
\hline & n.d & $\sim 10^{-5}$ & [92] \\
\hline & $3.4^{(\mathrm{d})}$ & n.d & [112] \\
\hline & $15 / 1.5^{\text {(d) }}$ & $7.6 \cdot 10^{-7}$ & {$[96,97]$} \\
\hline \multicolumn{4}{|l|}{ Photonic Crystals } \\
\hline & 0.42 & $3.4 \cdot 10^{-5}$ & [124] \\
\hline & 500 & $\sim 10^{-3}$ & {$[142,143]$} \\
\hline & $2.1^{(\mathrm{d})}$ & $6 \cdot 10^{-4}$ & {$[145]$} \\
\hline & $7.5^{(\mathrm{d})}$ & $7 \cdot 10^{-5}$ & {$[148,150]$} \\
\hline & 1000 & n.d & [153] \\
\hline \multicolumn{4}{|l|}{ Silicon Wires } \\
\hline Mach-Zehnder configuration & $0.25^{(\mathrm{d})}$ & n.d & [161] \\
\hline Resonator configuration & n.d & $<2 \cdot 10^{-6}$ & [162] \\
\hline \multicolumn{4}{|l|}{ Slot-waveguides } \\
\hline & 16 & n.d & {$[167]$} \\
\hline & n.d. & $8.8 \cdot 10^{-6}$ & [169] \\
\hline & 0.9 & $5 \cdot 10^{-6}$ & [168] \\
\hline & n.d & $4.2 \cdot 10^{-5}$ & [95] \\
\hline & n.d. & $7 \cdot 10^{-6}$ & [171] \\
\hline
\end{tabular}

${ }^{\mathrm{a}}$ :SPR is shown for comparison of the platforms with a standard optical biosensing device

b: averaged

c: n.d. not determined

d. estimated 
Table 2. Current commercial technologies based on integrated optics biosensing ${ }^{(a)}$

\begin{tabular}{|c|c|c|c|c|c|}
\hline Company & Instrument & Technology & Format & Throughput & Webpage \\
\hline Farfield & AnaLight & $\begin{array}{c}\text { Young } \\
\text { Interferometry }\end{array}$ & Flow conditions & Low & www.farfield-group.com \\
\hline SRU Biosensing & BIND Biosensor & Photonic Crystal & $\begin{array}{c}\text { Cartridge (16) } \\
\text { Microplate (96-1536) }\end{array}$ & Very High & www.srubiosystems.com \\
\hline Corning & EPIC System & $\begin{array}{c}\text { Waveguide } \\
\text { Resonant Grating }\end{array}$ & Microplate (384) & High & www.corning.com \\
\hline Axela & DotLab & Optical Grating & Flow/static conditions & Medium & www.axelabiosensors.com \\
\hline Microvacuum & OWLS & $\begin{array}{c}\text { Optical Grating } \\
\text { Couplers }\end{array}$ & Flow conditions & Low & www.owls-sensors.com \\
\hline Genalyte & Maverick & Ring resonators & $\begin{array}{c}\text { Consumable with } 1 \text { to } \\
128 \text { resonators }\end{array}$ & Medium & www.genalyte.com \\
\hline
\end{tabular}

${ }^{a}$ : Sensitivity levels are in the low $\mathrm{ng} / \mathrm{mL}$ range in all the cases. 